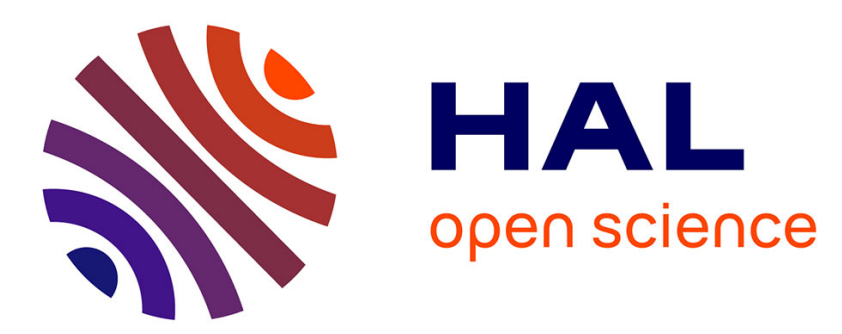

\title{
Dérivés du 4-spirobifluorène - Une nouvelle famille de matrices hôtes pour des PhOLED
}

Cyril Poriel, Joëlle Rault-Berthelot

\section{To cite this version:}

Cyril Poriel, Joëlle Rault-Berthelot. Dérivés du 4-spirobifluorène - Une nouvelle famille de matrices hôtes pour des PhOLED. Les Techniques de l'Ingenieur, 2017. hal-03388569

HAL Id: hal-03388569

https://hal-univ-rennes1.archives-ouvertes.fr/hal-03388569

Submitted on 20 Oct 2021

HAL is a multi-disciplinary open access archive for the deposit and dissemination of scientific research documents, whether they are published or not. The documents may come from teaching and research institutions in France or abroad, or from public or private research centers.
L'archive ouverte pluridisciplinaire HAL, est destinée au dépôt et à la diffusion de documents scientifiques de niveau recherche, publiés ou non, émanant des établissements d'enseignement et de recherche français ou étrangers, des laboratoires publics ou privés. 


\section{Dérivés du 4-Spirobifluorène en vue de leur utilisation comme matrices hôtes pour des OLEDs phosphorescentes}

\section{par Cyril PORIEL}

Docteur de l'Université de Rennes 1

CR1 CNRS, UMR CNRS 6226, Institut des Sciences Chimiques de Rennes

\section{et Joëlle RAULT-BERTHELOT}

\section{Docteur d'Etat en Chimie}

DR2 CNRS, UMR CNRS 6226, Institut des Sciences Chimiques de Rennes

Résumé: Les dérivés du 9,9'-Spirobifluorène (SBF) font partie des matériaux qui ont révolutionné l'électronique organique ces 20 dernières années. Tout particulièrement, l'incorporation de dérivés SBF (oligomères et/ou polymères) dans des Diodes Organiques Electroluminescentes (OLEDs) a permis de fortement améliorer leurs performances et stabilités. En effet, les dérivés du SBF présentent de très hautes stabilités thermiques, paramètre clé pour la stabilité des dispositifs électroniques. Les dérivés du SBF tiennent aussi une place de choix dans le domaine des cellules solaires organiques. Cependant, la quasi-totalité des dérivés du SBF synthétisés et étudiées dans la littérature sont substitués en position 2 du SBF (2-SBF), cette substitution en para permet une excellente délocalisation des électrons $\pi$ sur tout le motif fluorene et son substituant. Ainsi, les dérivés du SBF substitués sur d'autres positions (1-SBF, 3-SBF ou 4-SBF) sont quasiment absents de la littérature. Toutefois, récemment 17 dérivés 4-SBF ont été synthétisés, étudiés et utilisés comme matrices hôtes pour des OLEDs phosphorescentes (PhOLEDs). En effet, la substitution du SBF par un lien ortho permet de rompre efficacement la conjugaison (paramètre clé dans le design de matrices hôtes pour les PhOLEDs bleues) en gardant les propriétés physiques des dérivés SBF inchangées (haute stabilité thermique et morphologique). Cette revue a pour objectif de faire un état des lieux exhaustif de l'utilisation de ces nouveaux 4-SBF possédant un fort potentiel en tant que matrice hôte dans des PhOLEDs. Cette revue couvrira ainsi plusieurs aspects, après une approche générale sur la stratégie de synthèse des molécules 4-SBF, elle donnera une analyse détaillée et comparative de l'ensemble des propriétés (optiques, électrochimiques et thermiques) et mettra en exergue les applications des dérivés 4-SBF en tant que matrice hôte dans des PhOLEDs.

Abstract: 9,9'-Spirobifluorene (SBF) derivatives are materials which revolutionized the electronic organic these last 20 years. More precisely, the incorporation of SBF derivatives (oligomers and/or polymers) in organic light-emitting diodes (OLEDs) allowed to improve strongly their performances and stabilities. Indeed, SBF derivatives present very high thermal stability, key parameter for the stability of the electronic devices. SBF derivatives are also useful in the field of organic solar cells. Nevertheless, most of the mono-substituted-SBF synthesized and used in organic electronics are 
substituted at the carbon 2 of SBF (2-SBF), the para-linkage leading to a good $\pi$ delocalization on the substituted SBF and its substituent. Thus, SBF substituted at the other positions (1-SBF, 3-SBF or 4$\mathrm{SBF}$ ) are almost absent from literature. However, quite recently, 4-SBF derivatives make great progress as host material in phosphorescent organic light-emitting diodes (PhOLEDs). In fact, the SBF ortho-substitution at the carbon 4 leads to an efficient conjugation break between the substituted fluorene and its substituent (key parameter for the design of host materials for blue PhOLEDs) keeping however the other physical properties of SBF (high thermal and morphological stabilities). The aim of the present review is to present the state of the art of this recent family of 4-SBF molecules regarding mainly their physicochemical and photophysical properties and their applications as host in PhOLEDs.

Mots-clés: Spirobifluorène ortho-substitué, OLEDs phosphorescentes, Énergie de l'état triplet

Keywords: ortho-substituted SBF, Phosphorescent OLED, triplet state energy

\section{Points clés}

Domaine : Electronique organique, semi conducteurs organique

Degré de diffusion de la technologie : Emergence I Croissance I Maturité

Technologies impliquées : Diodes organiques phosphorescentes

Domaines d'application : Affichage / Eclairage

Principaux acteurs français :

Orgatech (LPICM, Ecole Polytechnique)

Elorga (Bordeaux)

Plateforme OLED 200 mm (CEA-LETI Grenoble)

Plateforme Organique IEMN (Villeneuve d'Ascq)

Plateforme Electronique Plastique (XLIM Limoges)

Pôles de compétitivité :

Minalogic, Tenerrdis,

Centres de compétence : Groupement de recherche en électronique organique (GDR 3368)

Industriels :

Autres acteurs dans le monde : LG, Siemens+Osram, Philips, Sony+Panasonic, Mitsubishi Electric, Verbatim, Konica Minolta, LG Chem, Inteltronic, DuPont Display, General Electric, Holst center.... 
Contact : Cyril Poriel - Joëlle Rault-Berthelot, Groupe MaCSE, UMR CNRS 6226 Institut des Sciences Chimiques de Rennes, 35042, France

cyril.poriel@univ-rennes1.fr,

joelle.rault-berthelot@univ-rennes1.fr 


\section{Contexte}

En 2015, la consommation globale d'électricité de l'éclairage en France est de 56 TWh, soit $12 \%$ de la consommation d'électricité française (475 TWh en 2015) (source ADEME 2013/2014). ${ }^{[1]}$ Depuis plusieurs dizaine d'années, le marché de l'éclairage a connu deux grandes évolutions grâce au développement des ampoules fluorescentes et à celui des diodes électroluminescentes (LED : lightemitting diode), ces deux voies ayant pour but de réduire la consommation électrique des sources d'éclairages. ${ }^{[2]}$ Bien que le rendement des LEDs puisse être encore amélioré, cette technologie présente des inconvénients et principalement un mauvais indice de rendu de couleur (IRC) dû à une émission monochromatique. Les composés organiques sont connus pour leur émission large, ils ont alors été intégrés comme couche active dans des OLEDs (Organic Light-Emitting Diode). Dès lors, une OLED fonctionne grâce à la fluorescence des molécules organiques de sa couche active. La mise sous tension d'une OLED entraine le départ d'un électron de la HOMO de la couche organique à l'anode et l'ajout d'un électron dans la LUMO de la couche organique coté cathode. Après migration, ces charges peuvent se recombiner et former un exciton, c'est-à-dire une molécule à l'état excité. La décorrélation des électrons, conduit à une distribution statistique entre les états singulets et triplets dans un ratio de 1 pour 3 . II y a alors uniquement $25 \%$ d'états excités singulets potentiellement fluorescents alors que $75 \%$ d'états excités triplets sont perdus en conversion interne. Les performances d'une OLED sont donc malheureusement plafonnées à $25 \%$ de conversion d'énergie électrique en énergie lumineuse ce qui est insuffisant pour de l'éclairage haute performance. Une voie possible d'amélioration des performances de ces dispositifs a été d'utiliser des molécules phosphorescentes comme dopant de la couche organique créant ainsi les diodes organiques phosphorescentes (PhOLED pour Phosphorescent Organic Light-Emitting Diodes). Dans une PhOLED, les excitons singulets et triplets sont générés dans une couche mixte composée de la matrice organique et du dopant phosphorescent (en général un complexe organométallique de l'iridium). Dans la matrice organique les excitons générés sous tension de fonctionnement du dispositif vont être transférés vers le dopant qui pourra émettre par phosphorescence, le rendement théorique interne d'une PhOLED pouvant alors atteindre $100 \%$. Afin d'obtenir les meilleures performances possibles de la PhOLED, il faut choisir le meilleur couple matrice organique /dopant phosphorescent. Les conditions d'un bon transfert des excitons de la matrice vers le dopant impliquent que les niveaux d'énergies des états singulets et triplets de la matrice soient respectivement supérieurs à ceux du dopant. La longueur d'onde de l'émission, donc la couleur émise, est directement liée au niveau triplet du dopant. Parmi les multiples matériaux phosphorescents, les complexes d'iridium (III)

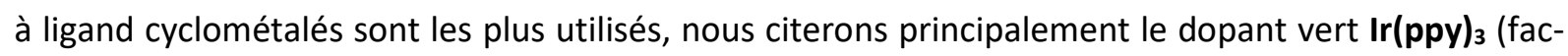
tris[2-phénylpyridinato- $\mathrm{C}^{2}, \mathrm{~N}$ ]iridium(III)) et le dopant bleu clair Flrpic (bis[2-(4,6-difluorophényl)pyridinato- $\left.\mathrm{C}^{2}, \mathrm{~N}\right]\left(\right.$ picolinato)iridium(III)). Ces deux dopants ont des états triplets de $2.44 \mathrm{eV}$ pour le vert ${ }^{[3]}$ 
et de $2.63 \mathrm{eV}$ pour le bleu ${ }^{[4]}$ nécessitant pour la matrice hôte associée un état triplet supérieur à 2.6 $\mathrm{eV}$ pour un bon transfert vers le dopant vert et supérieur à $2.8 \mathrm{eV}$ pour un bon transfert vers le dopant bleu. Si les matériaux hôtes adaptés aux dopants verts sont relativement courants aujourd'hui et conduisent à des PhOLEDs de bonnes performances, ${ }^{[5]}$ les matériaux hôtes adaptés aux dopants bleus et les dispositifs PhOLED émettant du bleu sont beaucoup plus rares. ${ }^{[6]}$ En effet, un matériau organique répondant au critère d'un état triplet de l'ordre de $2.8 \mathrm{eV}$ doit posséder un gap HOMO-LUMO proche de $4 \mathrm{eV}$ et est donc un matériau très faiblement conjugué. La recherche de matériaux hôtes à large gap HOMO-LUMO et à état triplet supérieur à $2.8 \mathrm{eV}$ est donc encore aujourd'hui un sujet majeur de la recherche dans le domaine des PhOLEDs.

Les stratégies utilisées aujourd'hui pour restreindre la conjugaison d'un matériau semi-conducteur organique (SCO) sont (i) l'utilisation de liens méta qui n'augmentent pas trop la conjugaison entre les différents systèmes $\pi$ conjugué, (ii) l'utilisation d'un lien via un hétéroatome $(\mathrm{Si}, \mathrm{P}, \ldots)$ qui isole les deux systèmes $\pi$ liés et enfin (iii) l'utilisation d'un pont spiro qui rompt la conjugaison entre les deux systèmes connectés. Enfin, plus récemment des ruptures de conjugaison par encombrement stérique ont été décrites. Ces gènes stériques conduisent à la diminution de la conjugaison entre deux systèmes $\pi$, l'encombrement empêchant l'aplanissement entre les systèmes $\pi$ connectés. Cette dernière stratégie est utilisée avec les SBF substitués en position ortho sur le carbone C4 du SBF et la littérature commence à présenter des performances de dispositifs PhOLEDs intéressantes en utilisant les 4-SBF comme matrice hôte en PhOLEDs bleues. C'est dans ce contexte que se situent les travaux présentés ci-dessous.

\section{Introduction}

Les composés d'architecture spiro dans lesquels au moins deux systèmes $\pi$ avec des propriétés identiques ou différentes sont connectés par un atome de carbone hybridé $\mathrm{sp}^{3}$ sont une classe importante de SCOs. ${ }^{[7]}$ Le 9,9'-spirobifluorene (SBF) est un des plus petits composés d'architecture $2 \pi-1$ spiro dans lequel deux unités fluorènes (diphényle ponté en C9) sont connectées via leurs ponts méthylènes par un unique atome de carbone (figure 1). Dans un composé $2 \pi$-1spiro, les deux unités sont dans deux plans perpendiculaires comme présenté figure 1 par la structure RX du SBF. ${ }^{[8]}$ Une telle architecture confère au SBF des propriétés intéressantes en termes de stabilités thermique, morphologique et de la couleur émise qui est une propriété clé dans les applications en OLED.

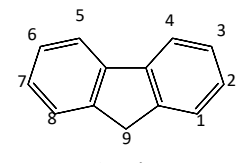

Fluorène
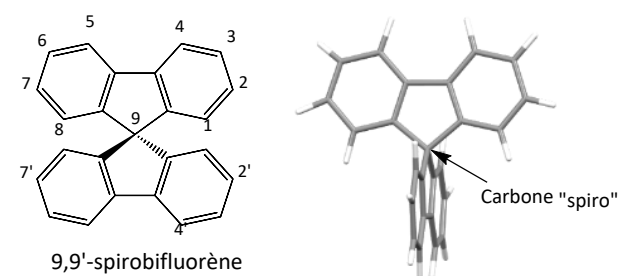

Figure 1: Fluorène et 9,9'-spirobifluorène (SBF), numérotation des différents atomes de carbone et structure $\mathrm{RX}^{\mathrm{du}} \mathrm{SBF}{ }^{[8]}$ 
Comme présenté figure 1, SBF possède 16 atomes de carbone potentiellement substituables (huit sur chaque unité fluorène), l'atome de carbone central « spiro » est lui non modifiable. Vu le nombre important de dérivés du SBF, nous nous concentrerons sur les SBF mono-substitués. Etant donné que la substitution électrophile du SBF se fait spécifiquement en position $2,{ }^{[7 a, 9]}$ les SBF substitués en position 2: 2-SBF, sont les plus nombreux dans la littérature laquelle ne rapporte que peu d'exemples de SBF substitués sur les positions 4 (4-SBF) ou 3 (3-SBF) et ne décrit que la synthèse du 1-méthyl-SBF. ${ }^{[10]}$

\section{Voie de synthèse générale aux 4-SBF}

Tous les dérivés du 4-SBF présentés dans cet article sont synthétisés à partir du 4-bromo-9,9'spirobifluorène (4-Br-SBF) ou de son dérivé pinacol borane (4-Pin-SBF) qui sont les plateformes centrales des voies de synthèse. 4-Br-SBF est obtenu en suivant deux voies d'accès présentées en figure 2, soit par couplage entre le 2,2'-dibromobiphényl 1 et la fluorénone $\mathbf{2}$ (Voie $A^{[11]}$ ) soit par couplage du 2-bromobiphényl 4 et la 4-bromo-fluorénone 5 (Voie $B^{[12]}$ ). Le rendement de formation du 4-Br-SBF à partir de $\mathbf{6}$ et $\mathbf{7}$ (Voie B) est de $45 \%$ alors que celui à partir de $\mathbf{1}$ et $\mathbf{2}$ (voie A) est de 83 \%. La voie B présente toutefois l'avantage de préparer la 4-bromo-fluorénone 5 qui est un intermédiaire clé pour la préparation d'autres dérivés du 4-SBF.
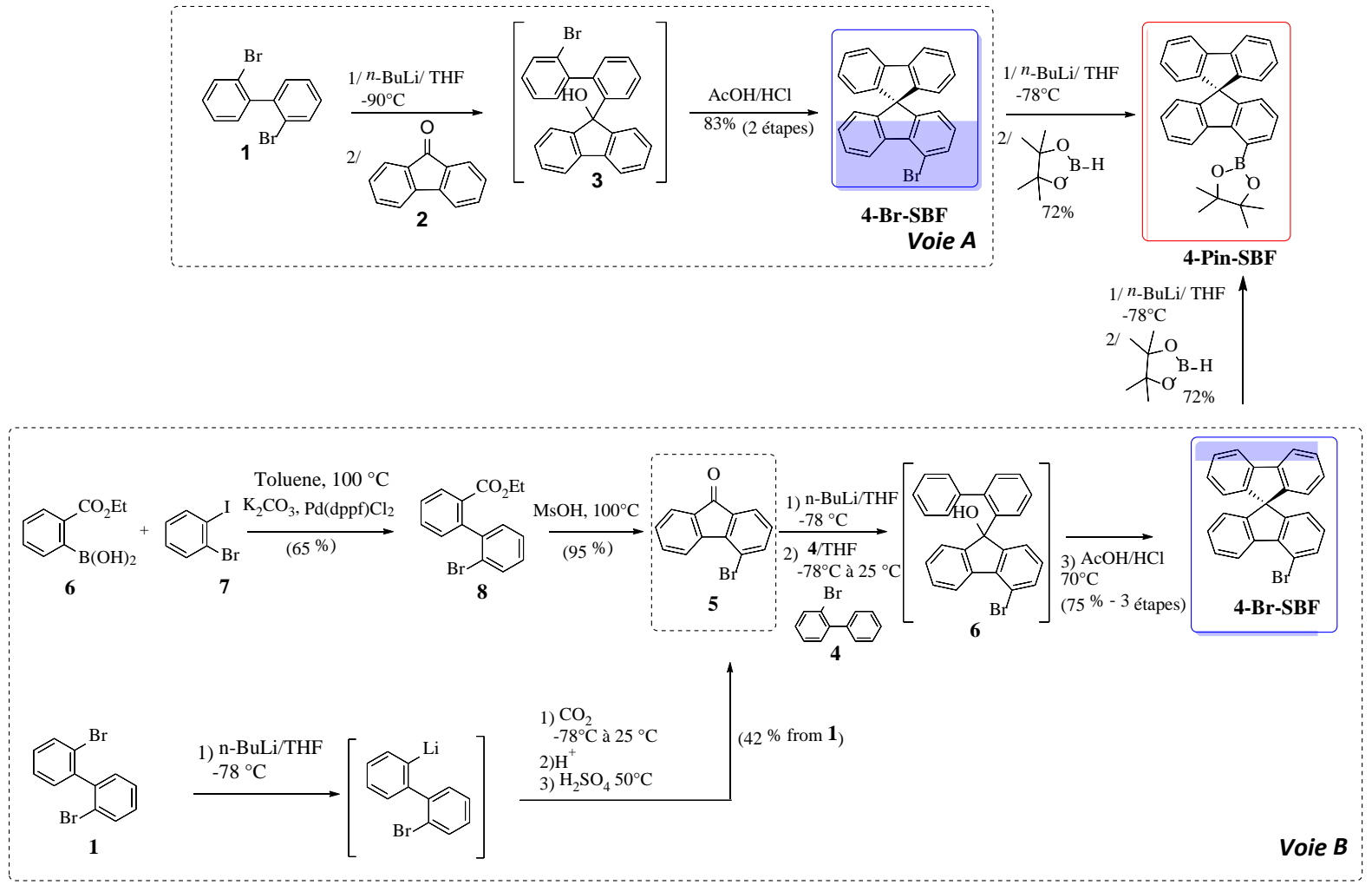

Figure 2. Les deux voies de synthèse de la brique centrale 4-Br-SBF et de son dérivé pinacol borane 4-Pin-SBF

Sans rentrer dans les détails de synthèse de chaque molécule présentées ci-dessous, la plupart ont été préparées par un couplage de Suzuki entre 4-Br-SBF (ou 4-Pin-SBF) et l'acide boronique (le dérivé 
bromé) du substituant d'intérêt de la molécule finale. Les synthèses du 4-Ph-SBF à partir du 4-Br-SBF et du 4-2Py-SBF à partir du 4-Pin-SBF sont présentées à titre d'exemple dans la figure 3, pour les autres molécules présentées dans le texte, le lecteur sera encouragé à lire les articles de référence.

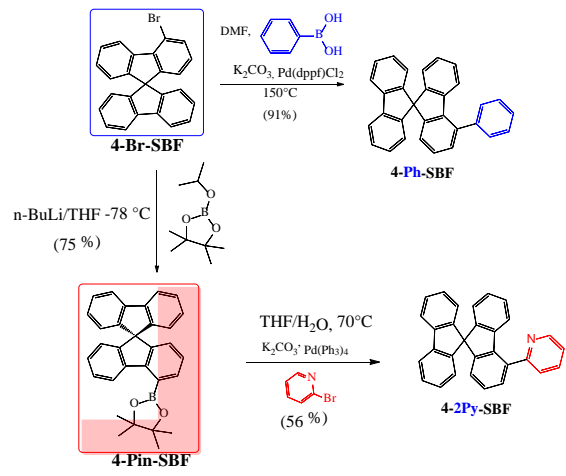

Figure 3. Synthèses du 4-Ph-SBF et du 4-2Py-SBF à partir du 4-Br-SBF ou du 4-Pin-SBF

\section{Origine de la rupture de conjugaison}

Contrairement aux dérivés SBF substitués en position 2 pour lesquels la conjugaison électronique peut s'étendre au substituant en position para du SBF, les dérivés substitués en position 4 appartiennent à une autre catégorie de SBF pour laquelle la conjugaison est interrompue entre le substituant en ortho du SBF et le SBF principalement du fait d'une gêne stérique. Cette rupture de conjugaison est décrite sur la figure 4 par la comparaison de la structure RX de cristaux de 4-Ph-SBF (à gauche) et de 2-Ph-SBF (à droite), chaque composé contient deux molécules indépendantes Mol 1 et Mol 2 par maille. ${ }^{[13]}$
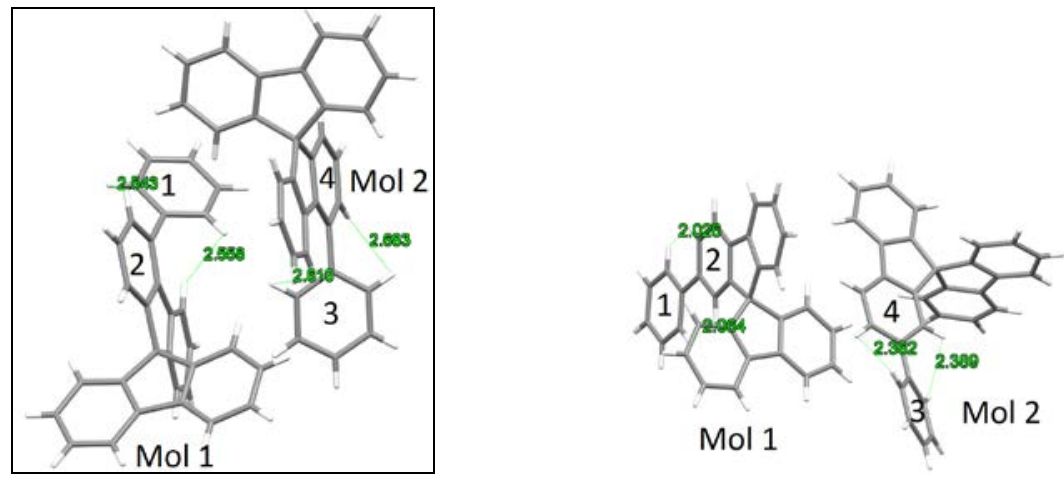

Figure 4. Structure RX de 4-Ph-SBF (gauche) et de 2-Ph-SBF (droite), chaque composé contient deux molécules indépendantes $\mathrm{Mol} 1$ et Mol 2 par unité.

Dans 2-Ph-SBF (figure 4, droite), l'angle entre le phényl 1 (ou 3) et le phényl du fluorène auquel il est lié ( 2 ou 4) est de $4.6^{\circ}$ dans $\mathrm{Mol} 1$ et de $37.4^{\circ}$ (dans Mol 2) maximisant la conjugaison entre les deux fragments. Dans les deux molécules, les distances entre les $\mathrm{H}$ en ortho du phényl et les $\mathrm{H}$ les plus proches des phényls 2 et 4 des fluorènes, comprises entre 2.06 at $2.37 \AA$, sont inférieures à la somme des rayons de Van der Waals de l'hydrogène (1.2 Å) montrant une interaction forte entre ces atomes. A l'opposé, dans 4-Ph-SBF (figure 4, gauche), l'angle entre Ph1 et Ph2 (Ph3 et Ph4 resp.) est de plus de $50^{\circ}\left(51.2^{\circ}\right.$ dans $\mathrm{Mol} 1$ ou $56.6^{\circ}$ dans Mol 2). L'augmentation sensible de cet angle est due à une 
gêne stérique entre les atomes d'hydrogène en position ortho du phényl 1 dans Mol 1 (ou 3 dans Mol 2) et les deux atomes d'hydrogène les plus proches de l'unité fluorène mis en évidence par les distances $\mathrm{H} / \mathrm{H}$ (comprises entre 2.54 et $2.67 \AA$ A) un peu plus importantes que celles mesurées dans 2Ph-SBF. Cette gêne stérique entraine aussi la déformation du fluorene portant le phényl avec un angle entre ses deux phényles de $12.71^{\circ}$ dans Mol 1 et de $4.77^{\circ}$ dans Mol 2 (dans SBF I'angle de déformation des deux fluorènes est de $1.26^{\circ}$ et $3.97^{\circ}$ et dans 2-Ph-SBF, la déformation du fluorène substitué est de $7.08^{\circ}$ dans $\mathrm{Mol} 1$ et de $1.5^{\circ}$ dans $\mathrm{Mol} 2$ ). La déformation des fluorènes substitués dans 4-Ph-SBF a pour origine les empilements intermoléculaires conduisant à de courtes distances $\mathrm{H} / \mathrm{H}$ (voire même $\mathrm{C} / \mathrm{H}$ ) qui entrainent une courbure de l'unité fluorène. En résumé, le lien ortho dans les 4-SBF a de fortes conséquences structurales qui auront elles-mêmes des répercussions importantes sur les propriétés électroniques. Principalement, du fait de la rupture de conjugaison entre le fluorène et son substituant dans 4-SBF, l'écart HOMO-LUMO reste aussi important que dans SBF et de ce fait le niveau de l'état triplet reste aussi élevé autorisant l'utilisation de la plupart des 4SBF comme matériaux hôtes pour les dopants bleus en PhOLEDs.

\section{4-SBF purement hydrocarbonés}

Dans cette première partie, nous avons répertoriés les dérivés du 4-SBF purement hydrocarbonés et comparerons leurs propriétés à celles de leurs homologues 2-SBF (quand ces homologues sont

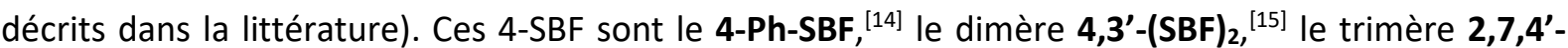
$(\mathrm{SBF})_{3}{ }^{[11]}$ et les oligomères $4,4^{\prime}-(\mathrm{SBF})_{n}$ (dimère, trimère, tétramère et pentamère) ${ }^{[11,16]}$.

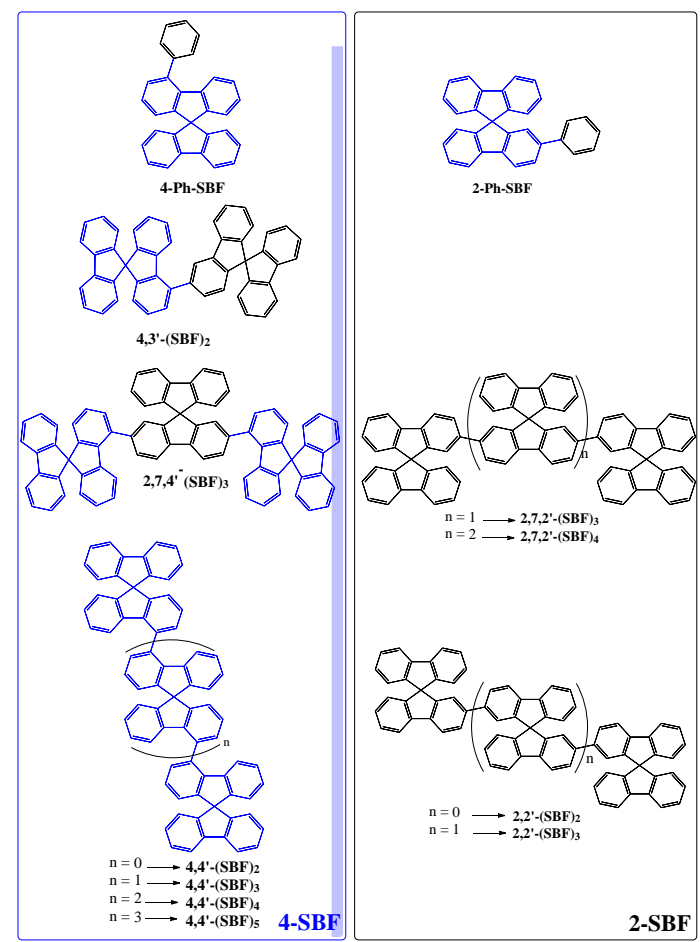

Figure 5. Composés hydrocarbonés dérivés du 4-SBF (gauche) ou du 2-SBF (droite). 
Concernant l'accès à ces molécules, elles ont été obtenues par couplage de Suzuki entre le 4-Br-SBF (respectivement le 2-Br-SBF ou leurs analogues pinacol (4-Pin-SBF ou 2-Pin-SBF)) et l'acide ou l'ester boronique adapté (voire le diacide dans le cas des oligomères). Les synthèses détaillées sont décrites dans les publications que le lecteur est invité à lire.

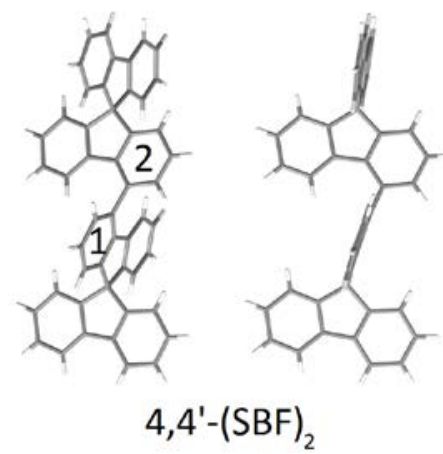

Figure 6. Structure RX du 4,4'-(SBF) $)_{2}$ vue sous deux angles différents ${ }^{[11]}$

Hormis le 4-Ph-SBF (resp. 2-Ph-SBF) dont la structure RX a été décrite et discutée dans la partie précédente (figure 4), seule la structure du dimère $4,4^{\prime}-(\mathrm{SBF})_{2}$ est décrite dans la littérature. ${ }^{[11]}$ Pour cette molécule, les auteurs annoncent un angle de $88.3^{\circ}$ entre les deux phényls ( 1 et 2 ) des unités fluorènes reliées en position $4 / 4^{\prime}$. Cet angle, encore plus important que celui mesuré entre le fluorène et le phényl dans 4-Ph-SBF ( $>51.2^{\circ}$ ) est proche de $90^{\circ}$ entrainant l'orthogonalité de chaque unité fluorène successive et la rupture de conjugaison entre chaque unité SBF (figure 6).

Les propriétés optiques des composés hydrocarbonés sont illustrées par la comparaison du couple SBF/4-Ph-SBF présenté figure 7.
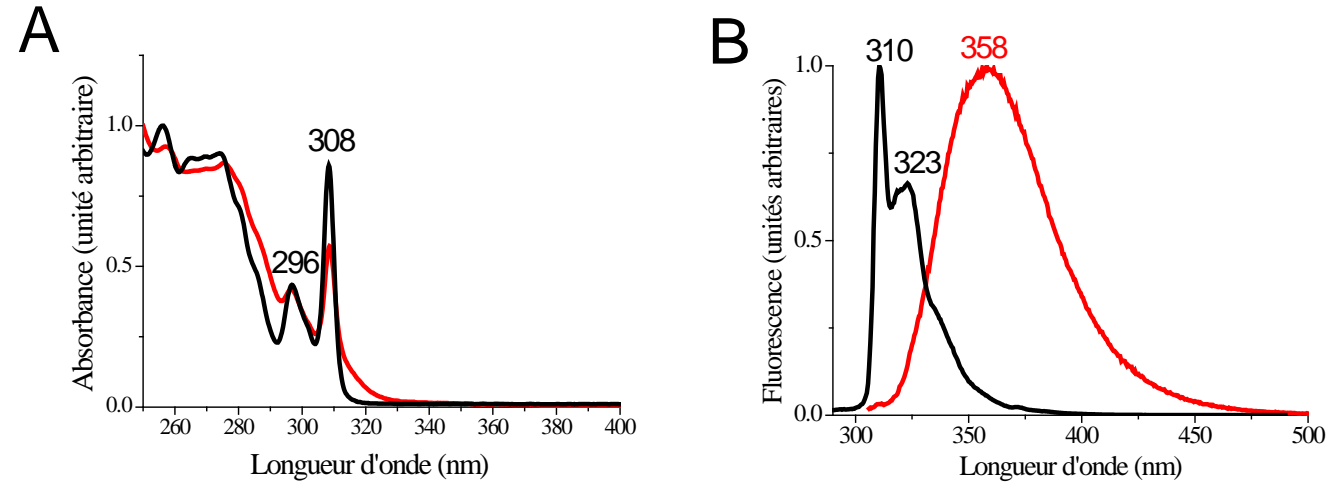

Figure 7. Spectres d'absorption (A) et d'émission de fluorescence (B) du 4-Ph-SBF (rouge) et du SBF (noir) enregistrés dans le cyclohexane, $\mathrm{C}=10^{-6} \mathrm{M}$.

Le spectre d'absorption du 4-Ph-SBF présente dans la zone de faible énergie deux bandes d'absorption dont les maxima sont à 296 et 308 nm, similaires aux maxima enregistrés pour le SBF ${ }^{[14]}$

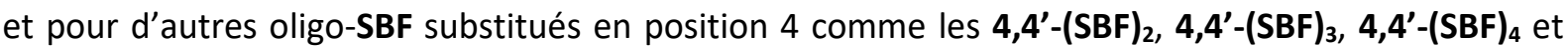
4,4'-(SBF) ${ }_{5}{ }^{[11,16]}$ Toutefois, pour 4-Ph-SBF, la bande d'absorption de plus faible énergie présente clairement une queue de bande trainant jusque $330 \mathrm{~nm}$, ce qui conduit à un gap HOMO-LUMO 
optique, $\Delta \mathrm{E}^{\mathrm{opt}}$, mesuré par la longueur d'onde de seuil d'absorption à $3.82 \mathrm{eV}$, plus contracté de 0.15 eV que celui du SBF (3.97 eV). Ainsi, bien que la conjugaison entre le fluorène et le phényl en position 4 du 4-Ph-SBF soit fortement restreinte par rapport à la conjugaison entre le fluorène et le phényl en position 2 du 2-Ph-SBF ( $\lambda_{\max }: 319 \mathrm{~nm}$ et $\Delta \mathrm{E}^{\text {opt }}: 3.7 \mathrm{eV}$ ), la queue de bande d'absorption observée pour 4-Ph-SBF est la signature de l'existence d'une faible conjugaison entre le phényl et le fluorène. Donc dans 4-Ph-SBF, la conjugaison n'est pas interrompue mais fortement restreinte. La rupture de conjugaison est par contre quasi-totale dans le cas des oligo-SBFs qui possèdent des $\Delta \mathrm{E}^{\text {opt }}$ de l'ordre de $3.93 \mathrm{eV},{ }^{[11,16]}$ similaires à celui du SBF $(3.97 \mathrm{eV})$ et ce quel que soit le nombre de motifs SBF liés. Ce comportement trouve son origine dans l'orthogonalité entre les unités SBF successives conduisant à une rupture totale de la conjugaison. L'encombrement dû au substituant en position 4 d'une unité SBF a donc une influence sur l'angle entre les deux unités liées $\left(>51^{\circ}\right.$ pour 4 -Ph-SBF et $>88^{\circ}$ pour $4,4^{\prime}$ $(\mathrm{SBF})_{2}$ ) et un effet important sur l'intensité de la conjugaison (plus l'angle est important et plus la rupture de conjugaison est forte).

Malgré une forte similarité des propriétés à l'état fondamental (similarité des spectres d'absorption), 4-Ph-SBF et SBF montrent des propriétés différentes à l'état excité, en effet leurs spectres d'émission présentés figure 7B sont très différents. 4-Ph-SBF présente un spectre d'émission large et non structuré $\left(\lambda_{\max }: 358 \mathrm{~nm}\right)$ alors que SBF présente un spectre résolu, image-miroir de son spectre d'absorption avec des maxima à 310 et $323 \mathrm{~nm}$. Une émission large et non structurée est aussi observée pour

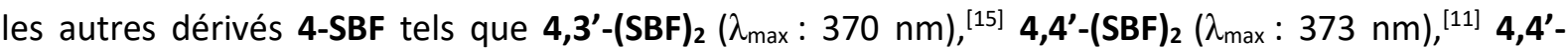

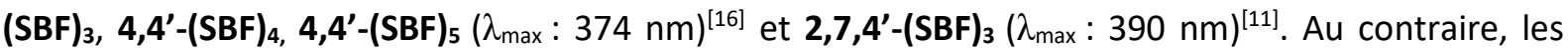
molécules 2-SBF $\left.\left(\mathbf{2}, \mathbf{2}^{\prime}-(\mathrm{SBF})_{2} \text { ou } \mathbf{2 , 2} \text { '-(SBF }\right)_{3}\right)^{[17]}$ présentent des spectres d'émission bien résolus signature du caractère de double liaison du lien $C=C$ entre les fluorènes entre eux à l'état excité. La perte de résolution des spectres d'émission est donc une caractéristique unique des composés 4-SBF non encore parfaitement élucidée dans la littérature. Le rendement de fluorescence $\left(\Phi_{\text {sol }}\right)$ du 4-Ph-SBF est reporté à 0.4 identique à celui du SBF et deux fois inférieur à celui du 2-Ph-SBF (0.87). L'augmentation importante du $\Phi_{\text {sol }}$ de 2-Ph-SBF par rapport au $\Phi_{\text {sol }}$ du 4-Ph-SBF s'explique par la combinaison d'une augmentation de la constante radiative $\left(k_{r} 5.610^{8} / 1.010^{6} \mathrm{~s}^{-1}\right.$ resp.) et d'une diminution de la constante non radiative ( $\mathrm{k}_{\mathrm{nr}} 8.310^{7} / 1.410^{8} \mathrm{~s}^{-1}$ resp.). Les oligo-SBFs 4,4'-(SBF),

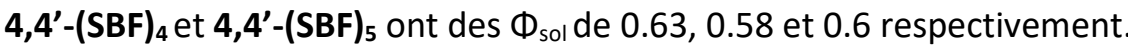

Pour pouvoir être utilisée comme matrice hôte pour un dopant phosphorescent, la matrice organique 4-SBF doit posséder un état triplet supérieur à celui du dopant afin d'assurer un transfert d'énergie efficace du matériau hôte vers le dopant. Les PhOLEDs émettrices de « bleu clair » utilisent généralement le FIrpic comme dopant, ce dernier a un état triplet à $2.62 \mathrm{eV} \cdot{ }^{[4]}$ Etant donné que les matrices organiques ne sont ordinairement pas phosphorescentes à température ambiante, la mesure de leur état triplet se fait à basse température $\left(-196{ }^{\circ} \mathrm{C} / 77 \mathrm{~K}\right)$ en enregistrant leur spectre 
d'émission dans une matrice gelée (mélange 1/1 de méthyl-cyclohexane/2-méthylpentane). L'état triplet est déterminé à partir de la longueur d'onde du pic d'émission de plus haute énergie. SBF présente ainsi un état triplet à $2.87 \mathrm{eV}\left(\lambda_{\max }: 431 \mathrm{~nm}^{[14]}\right)$ légèrement plus faible que celui du fluorène $(2.92 \mathrm{eV})$ indiquant une légère conjugaison entre les deux unités fluorènes du SBF à travers le pont spiro (spiroconjugaison). Les dérivés 4-SBF ont des $\mathrm{E}_{\mathrm{T}}$ proches de $2.8 \mathrm{eV}\left(2.76 \mathrm{eV}\right.$ pour $\mathbf{4 , 3} \mathbf{3}^{\prime}-(\mathrm{SBF})_{2}{ }^{[15]}$

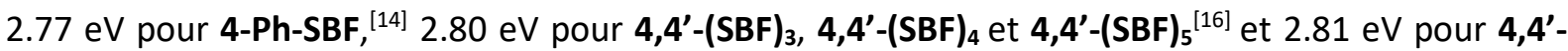
$\left.(\mathrm{SBF})_{2}{ }^{[11]}\right)$. Ces valeurs légèrement inférieures à celle du SBF montrent que la conjugaison n'est pas interrompue totalement entre le SBF et son substituant en C4 et que le nombre d'unités SBF liées dans les oligomères de type 4-SBF n'influence pas/peu la valeur de l'état triplet. En comparaison, les 2-SBF présentent des $E_{T}$ plus faibles (2.28 eV pour $\mathbf{2}^{\prime} \mathbf{2}^{\prime}-(\mathbf{S B F})_{3}, 2.3 \mathrm{eV}$ pour $\left.\mathbf{2}^{\prime} \mathbf{2}^{\prime}-(\mathbf{S B F})_{2}{ }^{[17]}\right)$, la délocalisation électronique induite par les liens para entre les unités fluorène diminue le bad et par voie de conséquence l'état triplet. L'encombrement stérique induit par la substitution en ortho des

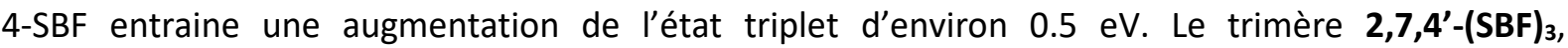
possédant à la fois des liens ortho et para, a un $\mathrm{E}_{\mathrm{T}}$ de $2.55 \mathrm{eV}^{[11]}$, intermédiaire entre celui du 4,4'-

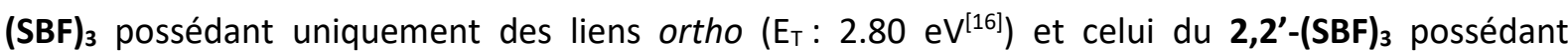
uniquement des liens para $\left(\mathrm{E}_{\mathrm{T}}: 2.28 \mathrm{eV}^{[17]}\right)$. Cet exemple illustre élégamment l'effet de la position de substitution sur les propriétés électroniques du trimère qui voit son $\mathrm{E}_{\mathrm{T}}$ baisser du fait des liens para du SBF central et l'augmentation de la conjugaison bien que celle-ci soit plus faible que dans $\mathbf{2 , 2}$ '$(\mathrm{SBF})_{3}$ du fait de l'encombrement stérique des SBF externes substitués en ortho. Des observations similaires ont aussi été faites pour les dimères de $S B F$ dont $E_{T}$ diminue de $2.81 \mathrm{eV}$ pour $\mathbf{4 , 4 ^ { \prime }}$-(SBF) ${ }_{2}{ }^{[11]}$

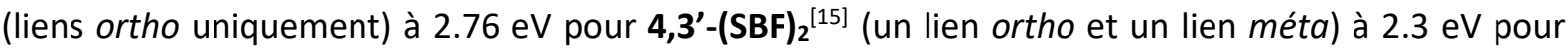
2,2'-(SBF) ${ }_{2}^{[17]}$ (liens para uniquement). Dans $4, \mathbf{3}^{\prime}-(\mathrm{SBF})_{2}$, le découplage électronique partiel induit par la substitution en méta couplé à l'encombrement stérique induit par la substitution en para conduit à un $E_{T}$ proche de celui du dimère à deux liens para $\left(4,4^{\prime}-(S B F)_{2}\right)$ montrant l'intérêt de cet arrangement moléculaire pour maintenir un $\mathrm{E}_{\mathrm{T}}$ élevé.

La stabilité thermique des matrices hôtes est aussi un paramètre important à prendre en compte avant toute intégration dans des dispositifs électroniques et particulièrement dans les OLEDs puisque ces dispositifs voient leur température augmenter par effet Joule en cours de fonctionnement, ces températures pouvant atteindre jusque $90^{\circ} \mathrm{C} .{ }^{[18]}$ La stabilité thermique des différents composants du dispositif électronique est donc essentielle pour éviter leurs décompositions au cours du fonctionnement. Deux paramètres thermiques principaux doivent être pris en compte: la température de décomposition $\left(T_{d}\right)$ mesurée par analyse thermogravimétrique et la température de transition vitreuse $\left(T_{g}\right)$ obtenue par calorimétrie différentielle à balayage. $T_{d}$ est définie par la température à laquelle le matériau étudié perd $5 \%$ de sa masse totale $\left(T_{d}\right.$ peut être vue comme la température de sublimation du matériau quand la perte de masse totale débute à cette 
température). SBF, 2-Ph-SBF et 4-Ph-SBF ont des $T_{d}$ de 234,238 et $254^{\circ} \mathbf{C}$ montrant que la substitution par un phényl augmente faiblement la stabilité thermique du SBF. ${ }^{[13]}$ En revanche, substituer SBF par une autre unité SBF entraine une augmentation sensible de la $T_{d}$ qui est de $360^{\circ} \mathrm{C}$

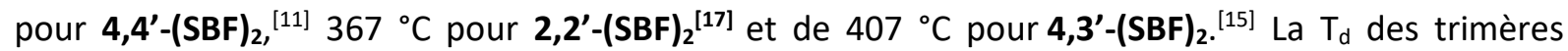

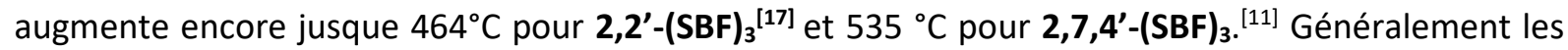
composés spiro sont caractérisés par une $\mathrm{T}_{\mathrm{g}}$ élevée indiquant la stabilité du matériau sous sa phase amorphe.

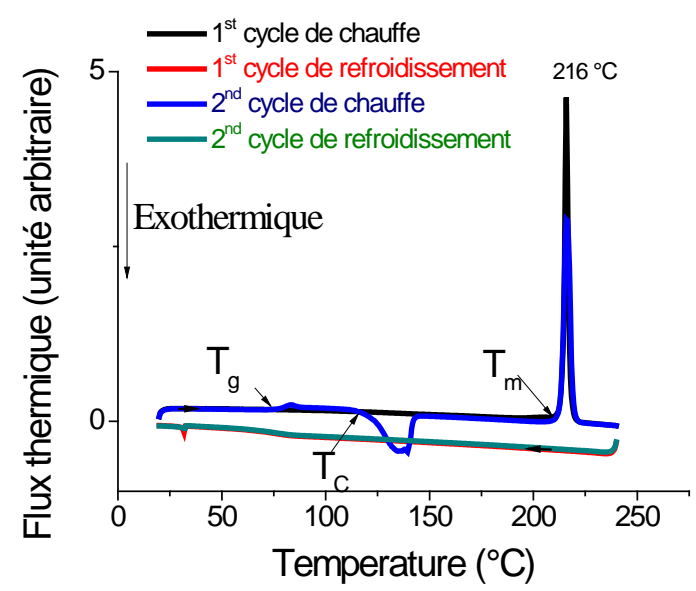

Figure 8. Thermogramme DSC du 4-Ph-SBF enregistré au cours de deux cycles successifs de chauffe/refroidissement.

La figure 8 présente à titre d'exemple le thermogramme DSC du 4-Ph-SBF enregistré au cours de deux cycles successifs de chauffe/refroidissement. 4-Ph-SBF présente au premier cycle de chauffe (courbe noire) un pic endothermique à $216^{\circ} \mathrm{C}$ associé à sa fusion (Température de fusion $\left(T_{m}\right)$ déterminée par le seuil du pic endothermique : $213^{\circ} \mathrm{C}$ ). Lorsque le liquide est refroidi à la même vitesse de 240 à $20^{\circ} \mathrm{C}$ (courbe rouge), aucune cristallisation n'est observée et le solide obtenu est amorphe. Au cours du second cycle de chauffe (courbe bleue), une transition vitreuse est observée vers $80^{\circ} \mathrm{C}\left(\mathrm{T}_{\mathrm{g}}\right.$ déterminée par le seuil du pic endothermique : $76^{\circ} \mathrm{C}$ ). En poursuivant la chauffe au-delà de $T_{g}$, un pic exothermique de cristallisation est observé au-delà de $139^{\circ} \mathrm{C}$ suivi d'un pic endothermique correspondant à la fusion du matériau cristallisé à $216^{\circ} \mathrm{C}$. Un tel comportement est typique de celui de la plupart des verres organiques. De ces thermogrammes, il a été démontré que 4-Ph-SBF présente une $\mathrm{T}_{\mathrm{g}}$ de $76^{\circ} \mathrm{C}$ similaire à celle du 2-Ph-SBF $\left(78^{\circ} \mathrm{C}\right) .{ }^{[13]}$ La substitution du SBF par une autre unité SBF conduit à une augmentation sensible de la $T_{\mathrm{g}}$ qui est de l'ordre de $170^{\circ} \mathrm{C}$ pour les dimères du $\mathrm{SBF}\left(170^{\circ} \mathrm{C}\right.$

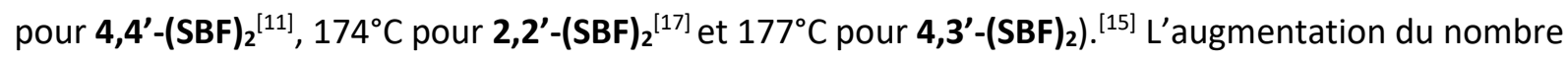
d'unités SBF liées entraine encore une augmentation de la $T_{d}$ qui est proche de $238^{\circ} \mathrm{C}$ pour les ter-

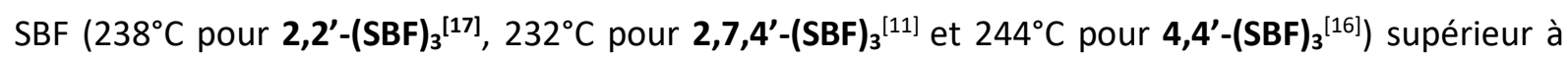

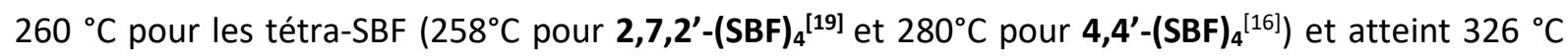
pour le penta-SBF $4,4^{\prime}-(\mathbf{S B F})_{5} \cdot{ }^{[16]}$ Si l'on observe que le nombre d'unités SBF a un impact important 
sur la valeur des $T_{\mathrm{g}}$ qui augmente sensiblement avec la longueur de l'oligomère, la position de la substitution n'a qu'une moindre influence sur ces valeurs.

Au bilan, on peut noter que les propriétés électroniques et particulièrement l'écart HOMO-LUMO et l'état triplet $\mathrm{E}_{\mathrm{T}}$ des quatre oligomères 4-SBF sont identiques $\left(\Delta \mathrm{E}^{\mathrm{opt}}: 3.93 \mathrm{eV}\right.$ et $\left.\mathrm{E}_{\mathrm{T}}: 2.8 \mathrm{eV}\right)$ alors que la stabilité thermique augmente sensiblement avec la longueur de l'oligomère $\left(T_{\mathrm{g}}\right.$ augmente de 170 à

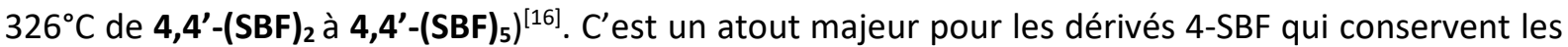
propriétés électroniques du SBF tout en améliorant ses propriétés physicochimiques.

Les propriétés physicochimiques des différentes molécules étudiées dans cette partie 3 ont été résumées dans le tableau 1.

Tableau 1. Propriétés physicochimiques des dérivés de SBF hydrocarbonés discutés dans la partie 3.

\begin{tabular}{|c|c|c|c|c|c|c|c|c|c|c|}
\hline & $\lambda_{\text {abs }}(\mathrm{nm})$ & $\begin{array}{l}\Delta E^{\text {opt }} \\
(\mathrm{eV})^{\mathrm{a}}\end{array}$ & $\begin{array}{l}\lambda_{\mathrm{PL}} \\
(\mathrm{nm})\end{array}$ & $\begin{array}{l}E_{\mathrm{T}} \\
(\mathrm{eV})\end{array}$ & QY & $\begin{array}{l}\text { HOMO } \\
(\mathrm{eV})^{\mathrm{b}}\end{array}$ & $\begin{array}{l}\text { LUMO } \\
(\mathrm{eV})\end{array}$ & $\begin{array}{l}\Delta E^{\mathrm{el}} \\
(\mathrm{eV})\end{array}$ & $\begin{array}{c}T_{g} \\
\left({ }^{\circ} \mathrm{C}\right) \\
\end{array}$ & $\begin{array}{c}T_{d} \\
\left({ }^{\circ} \mathrm{C}\right)\end{array}$ \\
\hline 4-Ph-SBF ${ }^{[14]}$ & $\begin{array}{l}257,275,296, \\
308 \text { (+ queue } \\
\text { de bande) }\end{array}$ & 3.82 & 358 & 2.77 & 0.4 & $-5.95^{b}$ & $-1.95^{b}$ & $4.00^{b}$ & 76 & 254 \\
\hline 2-Ph-SBF[13] & $397,308,319$ & 3.70 & 335,351 & 2.56 & 0.87 & $-5.88^{b}$ & $-2.10^{b}$ & $3.78^{b}$ & 78 & 238 \\
\hline SBF[14] & $\begin{array}{l}257,275,296, \\
308\end{array}$ & 3.97 & 310,323 & 2.87 & 0.40 & $-5.94^{b}$ & $-1.89^{b}$ & $4.05^{b}$ & no & 234 \\
\hline $4,4^{\prime}-(\mathrm{SBF})_{2}{ }^{[11]}$ & 309 & & 373 & 2.81 & & & & & 170 & 360 \\
\hline $4,4^{\prime}-(\mathrm{SBF})_{3}{ }^{[16]}$ & 308 & 3.93 & 374 & 2.80 & 0.63 & $-6.08^{b}$ & $2.15^{d}$ & & 244 & \\
\hline $4,4^{\prime}-(\mathrm{SBF})_{4}^{[16]}$ & 308 & 3.93 & 374 & 2.80 & 0.58 & $-6.08^{b}$ & $2.15^{d}$ & & 280 & \\
\hline $4,4^{\prime}-(\mathrm{SBF})_{5}{ }^{[16]}$ & 308 & 3.93 & 374 & 2.80 & 0.60 & $-6.08^{b}$ & $2.15^{d}$ & & 326 & \\
\hline $4,3^{\prime}-(\mathrm{SBF})_{2}{ }^{[15]}$ & 318 & 3.65 & 370 & 2.76 & & $-5.76^{c}$ & $2.11^{d}$ & & 177 & 407 \\
\hline $2,7,4^{\prime}-(\mathrm{SBF})_{3}{ }^{[11]}$ & $309 / 325$ & & 390 & 2.55 & & & & & 232 & 535 \\
\hline $2,2^{\prime}-(\mathrm{SBF})_{2}^{[17]}$ & 310,333 & & 367,387 & 2.3 & $0.66^{\mathrm{e}}$ & & & & 174 & 367 \\
\hline $2,2^{\prime}-(\mathrm{SBF})_{3}{ }^{[17]}$ & 310,333 & & 367,387 & 2.28 & $0.56^{\mathrm{e}}$ & & & & 228 & 464 \\
\hline $2,7,2^{\prime}-(\mathrm{SBF})_{3}{ }^{[19]}$ & 350 & & & & & & & & 237 & \\
\hline $2,7,2^{\prime}-(\mathrm{SBF})_{4}{ }^{[19]}$ & 360 & & & & & & & & 258 & \\
\hline
\end{tabular}

a $\Delta E^{\text {opt }}$ calculé à partir de la longueur d'onde de seuil d'absorption $\left(1239.84 / \lambda_{\text {seuil }}(\mathrm{nm})\right) ; \mathrm{b}$. à partir du potentiel de seuil d'oxydation ou de réduction de la voltammétrie cyclique ; c. à partir de spectrométrie photoélectronique UV ; d. LUMO $=\Delta E^{\text {opt }}-\mathrm{HOMO}$; e. mesuré avec une sphère d'intégration à l'état solide; no: non observé.

La plupart des 4-SBF présentés ci-dessus ayant un $\mathrm{E}_{\mathrm{T}}$ supérieur à $2.7 \mathrm{eV}$ ont été utilisés comme matrice hôte pour le dopant bleu Flrpic ( $\left.\mathrm{E}_{\mathrm{T}}: 2.63 \mathrm{eV}\right)$ ou pour le dopant vert Ir(ppy) ( $\left._{\mathrm{T}}: 2.44 \mathrm{eV}\right)$. La comparaison des performances des diodes entre elles peut être sujette à caution du fait de la différence des structures des diodes et de la façon de présenter les résultats dans la littérature. Nous regarderons donc ces comparaisons avec prudence en n'en ressortant que les tendances principales sur l'adaptation de la matrice au dopant et l'efficacité du transfert énergétique. Les principaux résultats des dispositifs PhOLEDs sont présentés dans le tableau 2.

4-Ph-SBF et SBF ont été utilisés comme matrice hôte pour le dopant vert $\operatorname{Ir}(\mathrm{ppy})_{3}$ dans des dispositifs d'architecture identique*: ITO/CuPc(10 nm)/NPB(40 nm)/TCTA(10 nm)/matrice hôte:Ir(ppy) ${ }_{3}(20$ $\mathrm{nm}) / \operatorname{TPBi}(40 \mathrm{~nm}) / \mathrm{LiF}(1.2 \mathrm{~nm}) / \mathrm{Al}(100 \mathrm{~nm}) \cdot{ }^{[14]}$ Avec 4-Ph-SBF comme matrice hôte, le dispositif émet de la lumière dès $3.5 \mathrm{~V}$ et atteint une efficacité en courant (CE) de $48.1 \mathrm{~cd} / \mathrm{A}$ et une puissance

\footnotetext{
* Les acronymes et le rôle des matériaux utilisés comme électrode ou couches intermédiaires dans les dispositifs électroniques sont donnés en fin du document
} 
énergétique $(P E)$ de $36 \mathrm{~lm} / \mathrm{W}$ équivalent à un rendement quantique externe (EQE) de $10.4 \%$. Un dispositif similaire utilisant SBF comme matrice hôte présente des performances légèrement moins élevée ( $C E=33.4 \mathrm{~cd} / \mathrm{A}, \mathrm{PE}=20.5 \mathrm{~lm} / \mathrm{W}$ et $\mathrm{EQE}=8.5 \%)$ avec toutefois un seuil de fonctionnement un peu plus faible $(3.1 \mathrm{~V})$. Au bilan, la substitution de SBF par un phényl en position 4 permet d'augmenter l'EQE du dispositif de presque $2 \%$.

Avec un $E_{T}$ de $2.55 \mathrm{eV}$, le trimère 2,7,4'-(SBF) 3 a été utilisé comme matrice hôte du dopant vert $\operatorname{Ir}(\text { ppy })_{3}$ en utilisant une diode d'architecture $1 \mathrm{TO} / \mathrm{MoO}_{3}(10 \mathrm{~nm}) / \mathrm{NPB}(40 \mathrm{~nm}) / \mathbf{2 , 7 , 4}-(\mathrm{SBF})_{3}: \operatorname{Ir}(\mathrm{ppy})_{3}$ $(30 \mathrm{~nm}) / \mathrm{BCP}(10 \mathrm{~nm}) / \mathrm{Alq}_{3}(30 \mathrm{~nm}) / \mathrm{LiF}(1 \mathrm{~nm}) / \mathrm{Al}(100 \mathrm{~nm}) \cdot{ }^{[11]}$ Avec un taux de dopage de $8 \%$ en masse, le dispositif atteint une CE de $48.2 \mathrm{~cd} / \mathrm{A}$, une PE de $26.8 \mathrm{Im} / \mathrm{W}$ soit un EQE de $12.6 \%$. Le trimère 4,4'$(\mathrm{SBF})_{3}$, dans lequel les trois $\mathrm{SBF}$ sont liés en ortho et possédant un $\mathrm{E}_{\mathrm{T}}$ de $2.80 \mathrm{eV}$ a été utilisé comme matrice hôte pour le même dopant vert dans un dispositif d'architecture voisine: ITO/ $\mathrm{MoO}_{3}(10$ $\mathrm{nm}) / \mathrm{NPB}(80 \mathrm{~nm}) / \mathrm{TCTA}(5 \mathrm{~nm}) / \mathbf{4 , 4}-\mathbf{- ( S B F})_{3}: \operatorname{Ir}(\text { ppy })_{3}(20 \mathrm{~nm}) / \operatorname{TPBi}(40 \mathrm{~nm}) / \mathrm{LiF}(1 \mathrm{~nm}) / \mathrm{Al}(120 \mathrm{~nm}) \cdot{ }^{[16]}$ Avec un taux de dopage de $11 \%$, EQE atteint $17.3 \%$ pour une CE de $66 \mathrm{~cd} / \mathrm{A}$ et une PE de $46 \mathrm{Im} / \mathrm{W}$. L'augmentation des performances peut être attribuée à la nature de la matrice qui possède un $E_{T}$ plus élevé, mais on ne peut négliger l'influence de la modification des couches intermédiaires des dispositifs (utilisation de $\mathrm{NPB}(80 \mathrm{~nm}) / \operatorname{TCTA}(5 \mathrm{~nm})$ au lieu de $\mathrm{NPB}(40 \mathrm{~nm})$ comme couche transporteur de trous et utilisation de TPBi $(40 \mathrm{~nm})$ à la place de BCP $(10 \mathrm{~nm}) / \mathrm{Alq}_{3}(30 \mathrm{~nm})$ comme couche de transporteur d'électron/bloqueur de trous). Au bilan des performances des PhOLEDs vertes, on notera les très bons résultats obtenus avec le trimère $4,4^{\prime}-(\mathrm{SBF})_{3}$ comme matrice hôte avec un EQE supérieur à $17 \%$ signant un transfert énergétique efficace entre la matrice et le dopant.

Table 2. Performances de diodes vertes ou bleues utilisant, comme matrice hôte, des dérivés de 4-SBF purement hydrocarboné.

\begin{tabular}{|c|c|c|c|c|c|c|}
\hline $\begin{array}{l}\text { Matrice Hôte } \\
E_{T}\end{array}$ & $\begin{array}{l}\text { Dopant } \\
\text { doping level }\end{array}$ & $V_{\text {on }}(\mathrm{V})$ & E.Q.E (\%) & CE (cd/A) & $\mathrm{PE}(\mathrm{Im} / \mathrm{W})$ & Ref \\
\hline \multicolumn{7}{|c|}{ PhOLEDs à émission verte } \\
\hline $\begin{array}{l}4,4^{\prime}-(\mathrm{SBF})_{3} \\
2.80 \mathrm{eV}^{\mathrm{b}} \\
\end{array}$ & $\begin{array}{l}\text { Ir(ppy) } \\
11 \text { wt } \%\end{array}$ & nd & $\begin{array}{l}17.3 \\
\text { (max value) }\end{array}$ & $\begin{array}{l}66 \\
\text { (max value) }\end{array}$ & $\begin{array}{l}46 \\
\text { (max value) }\end{array}$ & [16] \\
\hline $\begin{array}{l}\mathbf{2 , 7}, \mathbf{4}^{\prime}-(\mathrm{SBF})_{\mathbf{3}} \\
2.55 \mathrm{eV}^{\mathrm{a}}\end{array}$ & $\begin{array}{l}\operatorname{Ir}(\text { ppy })_{3} \\
8 \text { wt \% }\end{array}$ & 4 & $\begin{array}{l}12.6 \\
\text { (max value) }\end{array}$ & $\begin{array}{l}48.2 \\
\text { (max value) }\end{array}$ & $\begin{array}{l}26.8 \\
\text { (max value) }\end{array}$ & {$[11]$} \\
\hline $\begin{array}{l}\text { 4-Ph-SBF } \\
2.77 \mathrm{eV}^{\mathrm{c}}\end{array}$ & $\begin{array}{l}\text { Ir(ppy) } \\
9 \text { wt \% }\end{array}$ & $\begin{array}{l}3.5 \\
@ 1 \mathrm{~cd} / \mathrm{m}^{2}\end{array}$ & $\begin{array}{l}10.4 \\
@ 10 \mathrm{~mA} / \mathrm{cm}^{2}\end{array}$ & $\begin{array}{l}40.3 \\
@ 10 \mathrm{~mA} / \mathrm{cm}^{2}\end{array}$ & $\begin{array}{l}13.7 \\
@ 10 \mathrm{~mA} / \mathrm{cm}^{2}\end{array}$ & [14] \\
\hline $\begin{array}{l}\text { SBF } \\
2.87 \mathrm{eV}^{\mathrm{c}}\end{array}$ & $\begin{array}{l}\text { Ir(ppy) } \\
9 \text { wt } \%\end{array}$ & $\begin{array}{l}3.1 \\
@ 1 \mathrm{~cd} / \mathrm{m}^{2}\end{array}$ & $\begin{array}{l}8.5 \\
\text { (max value) }\end{array}$ & $\begin{array}{l}3.4 \\
\text { (max value) }\end{array}$ & $\begin{array}{l}20.5 \\
\text { (max value) }\end{array}$ & {$[14]$} \\
\hline \multicolumn{7}{|c|}{ PhOLEDs à émission bleu clair } \\
\hline $\begin{array}{l}4,3^{\prime}-(\mathrm{SBF})_{2} \\
2.76 \mathrm{eV}\end{array}$ & $\begin{array}{l}\text { FIrpic } \\
8 \text { wt \% }\end{array}$ & 3.0 & $\begin{array}{l}22 \\
\text { (max value) }\end{array}$ & $\begin{array}{l}44.5 \\
\text { (max value) }\end{array}$ & $\begin{array}{l}36.5 \\
\text { (max value) }\end{array}$ & {$[15]$} \\
\hline $\begin{array}{l}\text { 4-Ph-SBF } \\
2.77 \mathrm{eV}^{\mathrm{c}}\end{array}$ & $\begin{array}{l}\text { Flrpic } \\
19 \text { wt \% }\end{array}$ & $\begin{array}{l}4.0 \\
@ 1 \mathrm{~cd} / \mathrm{m}^{2}\end{array}$ & $\begin{array}{l}5.7 \\
@ 10 \mathrm{~mA} / \mathrm{cm}^{2}\end{array}$ & $\begin{array}{l}16.2 \\
@ 10 \mathrm{~mA} / \mathrm{cm}^{2}\end{array}$ & $\begin{array}{l}6.0 \\
@ 10 \mathrm{~mA} / \mathrm{cm}^{2}\end{array}$ & [14] \\
\hline $\begin{array}{l}4,4^{\prime}-(\mathrm{SBF})_{3} \\
2.80 \mathrm{eV}^{\mathrm{b}}\end{array}$ & $\begin{array}{l}\text { FIrpic } \\
8 \text { wt \% }\end{array}$ & nd & $\begin{array}{l}11.6 \\
\text { (max value) }\end{array}$ & $\begin{array}{l}25 \\
\text { (max value) }\end{array}$ & $\begin{array}{l}17 \\
\text { (max value) }\end{array}$ & {$[16]$} \\
\hline $\begin{array}{l}\text { SBF } \\
2.87 \mathrm{eV}^{\mathrm{c}}\end{array}$ & $\begin{array}{l}\text { Flrpic } \\
19 \text { wt \% }\end{array}$ & $\begin{array}{l}3.3 \\
@ 1 \mathrm{~cd} / \mathrm{m}^{2}\end{array}$ & $\begin{array}{l}6.6 \\
\text { (max value) }\end{array}$ & $\begin{array}{l}20.6 \\
\text { (max value) }\end{array}$ & nd & {$[14]$} \\
\hline
\end{tabular}

nd: non décrita ${ }^{\mathrm{A}}$ rchitecture du dispositif: $\quad \mathrm{ITO} / \mathrm{MoO}_{3}(10 \mathrm{~nm}) / \mathrm{NPB}(40 \mathrm{~nm}) /$ Matrice hôte-dopant $(30 \mathrm{~nm}) / \mathrm{BCP}(10 \mathrm{~nm}) / \mathrm{Alq}_{3}(30 \mathrm{~nm}) / \mathrm{LiF}(1$ $\mathrm{nm}) / \mathrm{Al}(100 \mathrm{~nm})$

${ }^{\mathrm{b}}$ Architecture du dispositif: ITO/MoO $3(10 \mathrm{~nm}) / \mathrm{NPB}(80 \mathrm{~nm}) / \mathrm{TCTA}(5 \mathrm{~nm}) /$ Matrice hôte-dopant $(20 \mathrm{~nm}) / \mathrm{TPBi}(40 \mathrm{~nm}) / \mathrm{LiF}(1 \mathrm{~nm}) / \mathrm{Al}(120 \mathrm{~nm})$ c Architecture du dispositif: ITO/CuPc(10 nm)/NPB(40 nm)/TCTA(10 nm)/Matrice hôte-dopant(20 nm)/TPBi(40 nm)/LiF(1.2 nm)/Al(100 nm) 
Les dispositifs PhOLED à émission bleu clair ont été testés avec trois matrices différentes $\mathbf{4 , 3}$ '-(SBF $)_{2}$ 4-Ph-SBF et 4,4'-(SBF) ${ }_{3}$, présentées par ordre de $E_{T}$ croissant de 2.76 à $2.80 \mathrm{eV}$, et comparés aux dispositifs utilisant SBF comme matrice hôte.

En comparant les matrices 4-Ph-SBF et SBF, la tendance observée avec les diodes vertes ne se retrouve pas avec les diodes bleues, en effet, malgré la similitude de l'architecture des dispositifs, la diode bleue à matrice SBF est plus efficace que celle avec 4-Ph-SBF. ${ }^{[13]}$ En effet, avec un taux de dopage de $19 \%$, le dispositif à matrice SBF éclaire à partir de $3.3 \mathrm{~V}$ et présente une $\mathrm{CE}$ de $20.6 \mathrm{~cd} / \mathrm{A}$ et une EQE de $6.6 \%$ alors que celle à base de 4-Ph-SBF n'éclaire qu'à partir de $4 \mathrm{~V}$ et présente une CE de $18.4 \mathrm{~cd} / \mathrm{A}$ et une EQE de $5.7 \%$. Malgré des états triplet quasi-équivalents, le transfert énergétique entre la matrice et le matériau hôte se fait moins efficacement avec 4-Ph-SBF qu'avec SBF.

La meilleure performance reportée dans la littérature par l'équipe de Liao, Jiang et al., ${ }^{[15]}$ a été obtenue avec le dimère ortho/méta 4,3'-(SBF) $)_{2}\left(\mathrm{E}_{\mathrm{T}}: 2.76 \mathrm{eV}\right)$ en utilisant le dispositif ITO/HAT-CN(10 $\mathrm{nm}) / \operatorname{TAPC}(40 \mathrm{~nm}) / \mathbf{4}, \mathbf{3}^{\prime}-(\mathrm{SBF})_{2}: \operatorname{Flrpic}(8 \mathrm{wt} \%)(20 \mathrm{~nm}) / \operatorname{TmPyPB}(45 \mathrm{~nm}) / \mathrm{Liq}(2 \mathrm{~nm}) / \operatorname{Al}(120 \mathrm{~nm})$. Avec ce dispositif, les auteurs annoncent une EQE de $22 \%$ pour une diode émettant dès $3 \mathrm{~V}$ et atteignant une CE de $44.5 \mathrm{~cd} / \mathrm{A}$ et une PE de $36.5 \mathrm{~lm} / \mathrm{W}$. Ces performances sont meilleures que celles reportées pour une diode bleue utilisant le trimère tout ortho $4,4^{\prime}-(\mathrm{SBF})_{3}{ }^{[16]}$ de $E_{\mathrm{T}}$ plus élevé (2.8 eV) en utilisant le dispositif ITO/ $\mathrm{MoO}_{3}(10 \mathrm{~nm}) / \mathrm{NPB}(80 \mathrm{~nm}) / \operatorname{TCTA}(5 \mathrm{~nm}) /$ 4,4'-(SBF) $)_{3}: \operatorname{Frpic}(8 \mathrm{wt} \%)(20 \mathrm{~nm}) / \mathrm{TPBi}(30$ $\mathrm{nm}) / \mathrm{LiF}(1 \mathrm{~nm}) / \mathrm{Al}(120 \mathrm{~nm})$. Les performances maximales sont de $25 \mathrm{~cd} / \mathrm{A}$ et $17 \mathrm{Im} / \mathrm{W}$ correspondant à une EQE de 11.6 \%. Là encore la comparaison entre les deux derniers dispositifs ne peut pas être attribuée uniquement à l'efficacité du transfert énergétique entre la matrice et le dopant mais il faut aussi considérer la différence entre les deux architectures des diodes.

Au bilan, les résultats obtenus avec les matrices purement hydrocarbonées dérivées du SBF ont permis de mettre en évidence l'intérêt de la substitution de ce dernier en position 4 à la fois pour améliorer les propriétés électroniques, la stabilité thermique et conduire à des dispositifs PhOLED pouvant atteindre des EQE de plus de $17 \%$ pour un dopant vert $\operatorname{Ir}(\mathrm{ppy})_{3}$ et de plus de $22 \%$ pour le dopant bleu FIrpic alors que les dispositifs à base de SBF ont des EQE de $8.5 \%$ pour le dopant vert et de $6.6 \%$ pour le dopant bleu.

Une voie d'amélioration des performances des dispositifs est de faciliter l'injection des charges dans la matrice hôte afin de pouvoir mettre en forme des dispositifs plus simples par exemple sans couche d'injection d'électrons ou de trous. La possibilité de modifier le corps SBF par des substituants accepteurs ou donneurs d'électrons sans modifier le gap HOMO-LUMO de la molécule et donc sans modifier le niveau de l'état triplet est une solution envisageable. Les deux parties suivantes traiteront donc des dérivés 4-SBF incorporant une unité déficitaire en électrons (partie 4) ou incorporant une unité riche en électron (partie 5). 


\section{4-SBF à substituant accepteur d'électrons}

II n'existe que peu d'exemples de dérivés 4-SBF à groupe déficitaire en électrons et nous ne décrivons ici que ceux ayant été utilisés comme matrice hôte dans des PhOLEDs. Ces six dérivés sont présentés figure 9. 4-5Pm-SBF, ${ }^{[13]}$ 4-4Py-SBF, 4-3Py-SBF et ${ }^{4-2 P y-S B F}{ }^{[12]}$ possèdent une fonction pyrimidine ou pyridine en position 4 du SBF. Leurs propriétés seront comparées à celles de leurs isomères dérivés du 2-SBF (2-5Pm-SBF, ${ }^{[13]}$ 2-4Py-SBF $\left.{ }^{[12]}\right)$. 4-POPh ${ }_{2}-\mathrm{SBF}$ décrit par Lee et al., ${ }^{[20]}$ en 2010 possède en position 4 du SBF l'unité diphénylphosphine oxyde $\mathbf{P O P h}_{2}$, son comportement sera comparé à celui de son isomère 2-POPh ${ }_{2}-\mathbf{S B F}^{[21]}$ Le dernier exemple, 2,7-DiCbz-SBF-4'-POPh ${ }_{2}^{[22]}$ comporte deux unités carbazoles $\mathrm{Cbz}$ en position 2 et 7 du fluorène lié via un carbone spiro au second fluorène portant une unité $\mathbf{P O P h}$ en position 4.

La synthèse des dérivés SBF à fonctions pyrimidine ou pyridine se fait par un couplage de Suzuki à partir des briques 4-Br-SBF ou 2-Br-SBF ou de leurs analogues pinacol (4-Pin-SBF ou 2-Pin-SBF)) et l'acide ou l'ester boronique adapté à la molécule finale. L'accès à 4-POPh ${ }_{2}-\mathbf{S B F}$ (ou à 2-POPh ${ }_{2}-\mathbf{S B F}$ ), se fait par lithiation du 4-Br-SBF (2-Br-SBF) suivie d'un piégeage de l'intermédiaire lithié par la chlorodiphénylphosphine $\left(\mathrm{ClPPh}_{2}\right)$. Le 4-PPh ${ }_{2}-\mathrm{SBF}\left(2-\mathrm{PPh}_{2}-\mathrm{SBF}\right)$ obtenu est alors oxydé par $\mathrm{H}_{2} \mathrm{O}_{2}$. En ce qui concerne 2,7-DiCbz-SBF-4'-POPh , il implique la synthèse du 2,7-DiCbz-SBF-4'-Br par réaction de la 2,7-DiCbz-fluorénone sur le 2,2'-dibromobiphényl puis la cyclisation intramoléculaire de l'alcool obtenu. L'accès à la molécule finale se fait selon le même processus que celui donnant accès à 4$\mathrm{POPh}_{2}$-SBF ou 2-POPh${ }_{2}$-SBF décrit ci-dessus (lithiation puis piégeage par $\mathrm{ClPPh}_{2}$ et oxydation par $\mathrm{H}_{2} \mathrm{O}_{2}$.

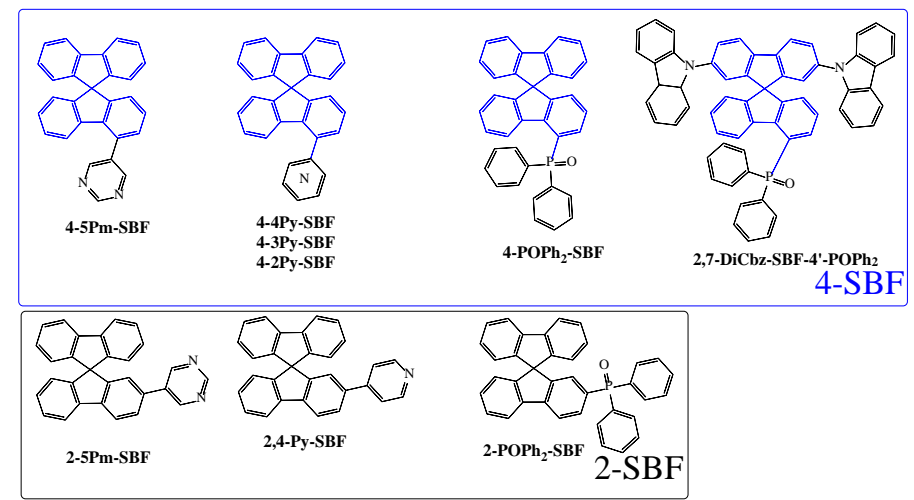

Figure 9. Composés dérivés du 4-SBF (en haut) ou du 2-SBF (en bas).

Concernant l'analyse structurale de ces molécules, elle n'est accessible que pour 4-4Py-SBF et 2,7diCbz-SBF-4'-POPh ${ }_{2}$ (ainsi que pour 2-4Py-SBF et 2-5Pm-SBF), les autres molécules n'ayant pas cristallisé (figure 10). De ces structures RX, on notera un angle de $42.2^{\circ}$ entre la pyridine et le phényl 1 du fluorène dans 4-4Py-SBF, plus important que ceux mesurés pour 2-4Py-SBF $\left(37.5^{\circ}\right.$ dans Mol 1 et $32.8^{\circ}$ dans $\mathrm{Mol} 2$ ). Là encore, cet angle plus important s'accompagne de distances entre les atomes d'hydrogène en ortho de la pyridine et ceux du phényl 1 du fluorène plus importante dans 4-4Py-SBF 
(2.44 et $2.61 \AA ̊$ ) que dans 2-4Py-SBF (2.27 et $2.4 \AA ̊$ Mol 1 et 2.39 et $2.42 \AA ̊$ dans Mol 2). La différence entre les angles pyridine/phényl 1 dans 4-4Py-SBF et 2-4Py-SBF $\left(9.4{ }^{\circ}\right.$ ) est plus faible que celle mesurée dans la série 4-Ph-SBF/2-Ph-SBF (autour de $13.8^{\circ}$ ) montrant que la nature du substituant joue aussi un rôle clé sur la valeur de cet angle qui va gérer les propriétés électroniques des composés. Nous verrons en effet que la diminution de l'angle (supérieur à $51^{\circ}$ dans $\mathbf{4}$-Ph-SBF et $42.2^{\circ}$ dans 4-4Py-SBF) s'accompagne d'une augmentation de la conjugaison entre la pyridine et le fluorène auquel elle est liée dans 4-4Py-SBF par rapport à celle entre le phényl et le fluorène dans 4Ph-SBF. Une plus forte déformation du fluorène substitué ( $16.8^{\circ}$ entre les deux phényl) est mesurée pour 4-4-Py-SBF (déformation $12.7^{\circ}$ dans 4-Ph-SBF et déformations inférieures à $5^{\circ}$ dans $2-4 \mathbf{P y}$-SBF).
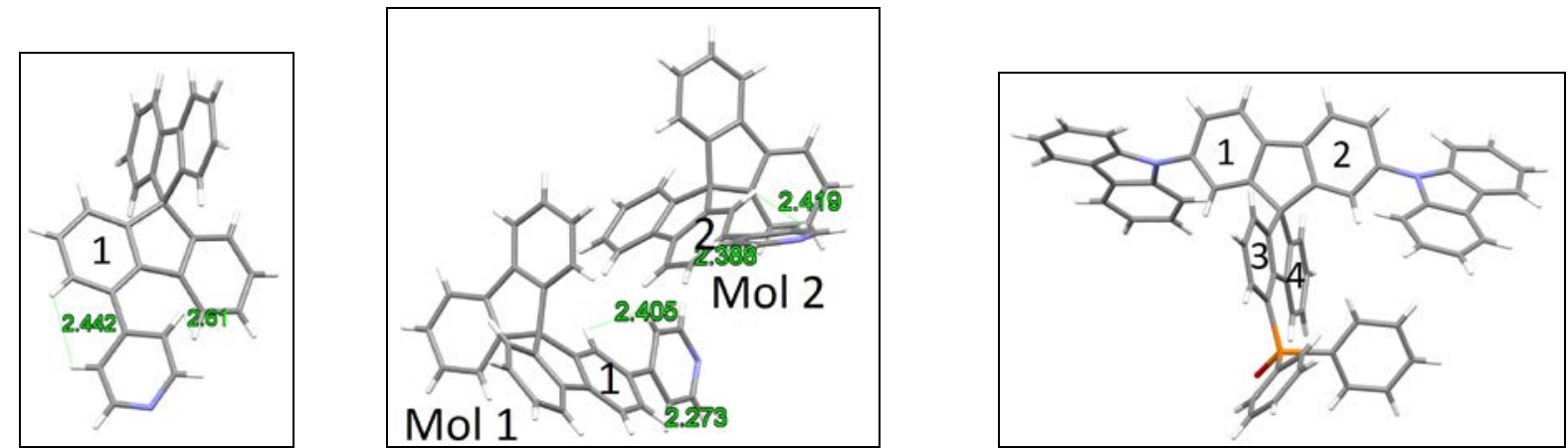

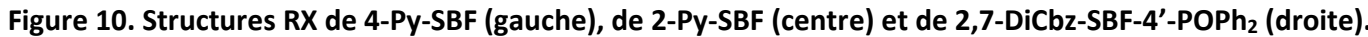

Dans 2,7-diCbz-SBF-4'-POPh , la déformation des deux unités fluorène n'est pas négligeable (angle de $8.3^{\circ}$ entre les plans des phényl 1 et 2 et angle de $7.3^{\circ}$ entre les phényls 3 et 4 ). Contrairement aux molécules 4-SBF précédentes, pour lesquelles la rupture de conjugaison était induite par la gêne stérique entre le fluorène et son substituant, dans 2,7-DiCbz-SBF-4'-POPh ${ }_{2}$ la rupture de conjugaison se fait via l'atome de phosphore du groupe $\mathrm{POPh}_{2}$.
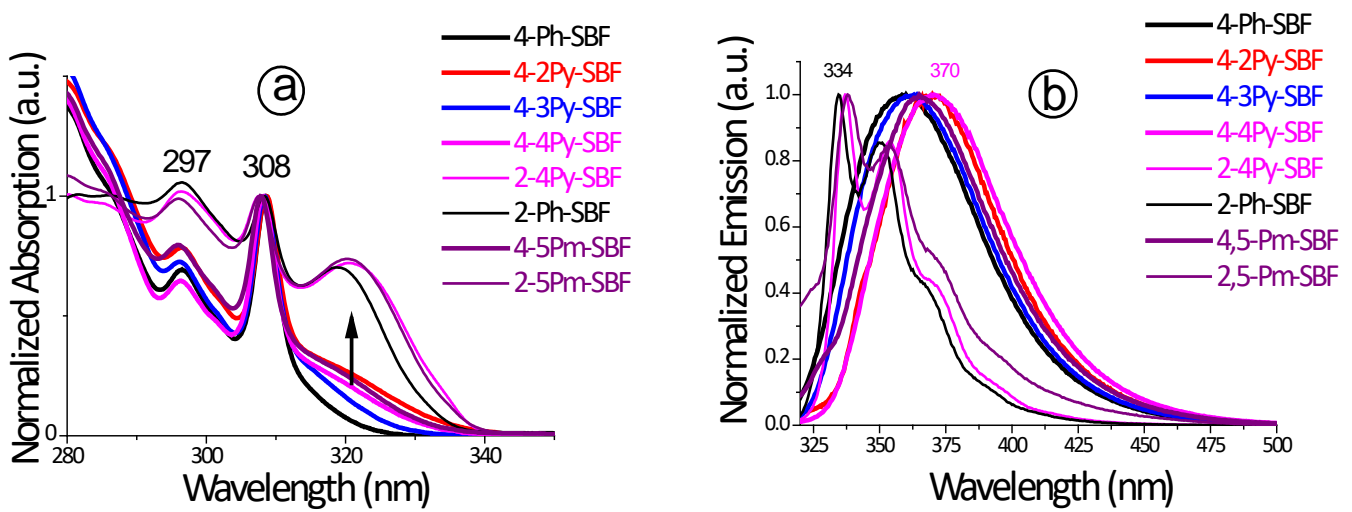

Figure 11. Spectres d'absorption (a) et d'émission de fluorescence (b) des 4-Ph-SBF et 2-Ph-SBF (noir), 4-2Py-SBF (rouge), 4-3Py-SBF (bleu), 4-4Py-SBF et 2-4Py-SBF (magenta) et 4-5Pm-SBF et 2-5Pm-SBF (violet) enregistrés dans le cyclohexane, $\mathrm{C}=10^{-6} \mathrm{M}$.

Comme présenté figure 11a, les 4-SBF substitués par une unité pyridine ou pyrimidine présentent des spectres d'absorption très similaires entre eux et à celui du 4-Ph-SBF, avec deux maxima à 297 et 
$308 \mathrm{~nm}$ et une queue de bande s'étendant jusque $340 \mathrm{~m}$. L'intensité de cette queue de bande varie avec la nature du groupement substituant et signe l'intensité de la conjugaison entre le fluorène et I'unité qui le substitue. La conjugaison maximale est observée pour 4-2Py-SBF et 4-5Pm-SBF puis 44Py-SBF et 4-3Py-SBF montrant que la position de l'atome d'azote a aussi une influence sur l'intensité de la conjugaison. Ce comportement est en accord avec la valeur de l'angle entre le fluorène et son substituant mesuré via les structures RX figure 10 qui apparait plus faible pour 4-4PySBF $\left(42.2^{\circ}\right)$ que pour 4-Ph-SBF $\left(51.2^{\circ}\right)$. Les $\Delta \mathrm{E}^{\text {opt }}$ mesurés à partir des spectres UV varient de $3.72 \mathrm{eV}$ pour 4-5Pm-SBF à $3.81 \mathrm{eV}$ pour 4-3Py-SBF et sont tous inférieurs à celui du 4-Ph-SBF (3.82 eV), montrant une extension de la conjugaison dans les 4-SBF substitués par des unités pauvres en électrons. La diminution du $\Delta \mathrm{E}^{\mathrm{opt}}$ est principalement due à la diminution de la LUMO des molécules à substituant électrodéficient. Cette augmentation de la conjugaison est aussi observée dans la série des 2-SBF avec une bande d'absorption supplémentaire centrée vers 319/320 nm dans la zone de faible énergie du spectre d'absorption. L'électrochimie est une technique qui permet d'étudier les propriétés électroniques des molécules (en oxydation et/ou en réduction) et de déterminer via l'enregistrement de voltammétries cycliques (VC) les niveaux HOMO, LUMO et donc l'écart HOMO/LUMO des molécules (aussi nommé $\Delta \mathrm{E}^{\mathrm{e}}$ ). Les figures 12 présentent les $\mathrm{CVs}$ des 4-4Py-SBF et 4-5Pm-SBF comparées à celle du 4-Ph-SBF.
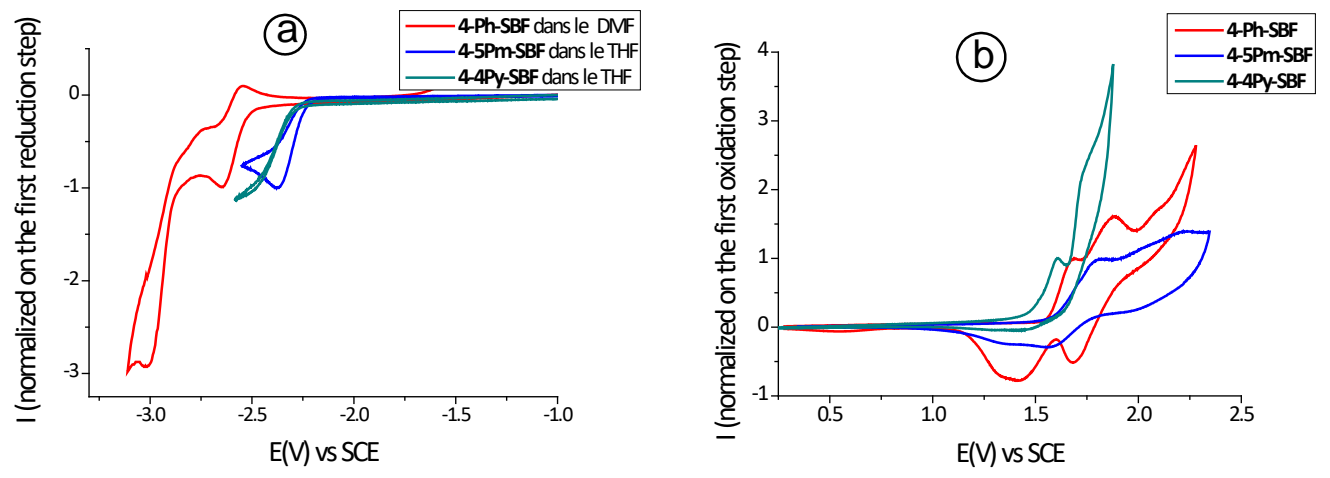

Figure 12. Voltammétries cycliques enregistrées : (a) en réduction (DMF ou THF $+0.1 \mathrm{M} \mathrm{Bu}_{4} \mathrm{NPF}_{6}$ ) et (b) en oxydation $\left(\mathrm{CH}_{2} \mathrm{Cl}_{2}+0.2 \mathrm{M} \mathrm{Bu}_{4} \mathrm{NPF}_{6}\right)$ de 4-Ph-SBF (rouge), 4-5Pm-SBF (bleu) and 4-4Py-SBF (vert). Les courants sont normalisés sur le premier pic d'oxydation ou de réduction. (Electrode de travail : disque de Platine $\emptyset 1 \mathrm{~mm}, 100 \mathrm{mV} / \mathrm{s}, \mathrm{CH}_{2} \mathrm{Cl}_{2}$ ).

La première oxydation de 4-4Py-SBF et celle de 4-5Pm-SBF ont lieu dans la même zone de potentiel que la première oxydation de 4-Ph-SBF $(1.7 \mathrm{~V} \pm 0.1 \mathrm{~V})$. Le greffage des unités pauvres en électrons en position 4 du corps SBF n'a donc que peu d'influence sur l'oxydation de ces composés et les HOMO déterminées à partir des potentiels de seuil d'oxydation sont de $-5.88 \mathrm{eV}$ pour les trois dérivés 4-PySBF et de -5.97 eV pour le 4-5Pm-SBF valeurs proches de la HOMO du 4-Ph-SBF (-5.95 eV). A l'opposé, la réduction électrochimique des 4-4Py-SBF et 4-5Pm-SBF a lieu à des potentiels moins négatifs (-2.48 et -2.37 V resp.) que celle du 4-Ph-SBF (-2.64 eV). Les valeurs des LUMO déterminées à partir des VCs sont de -2.11eV pour 4-4Py-SBF, -2.1 eV pour 4-2-Ph-SBF, -2.01 eV pour 4-3Py-SBF et - 
$2.23 \mathrm{eV}$ pour 4-5Pm-SBF fortement plus basses que la LUMO de 4-Ph-SBF mesurée à $-1.95 \mathrm{eV}$. La diminution de la LUMO induite par le cycle pyridine est moins prononcée que celle induite par la pyrimidine signant la différence de la force électroattractrice des deux hétérocycles. Les $\Delta \mathrm{E}^{\mathrm{el}}$ mesurés à partir des VCs varient de $3.74 \mathrm{eV}$ pour 4-5Pm-SBF à $3.89 \mathrm{eV}$ pour 4-3Py-SBF et sont tous inférieurs à celui du 4-Ph-SBF (4.0 eV), montrant une extension de la conjugaison dans les 4-SBF substitués par des unités pauvres en électrons. Cette variation des $\Delta \mathrm{E}^{\mathrm{el}}$ suit la même tendance que celles des $\Delta \mathrm{E}^{\mathrm{opt}}$.

Comme indiqué dans la partie 3, les dérivés 4-SBF présentent une émission large et non structurée avec des maxima mesurés à $370 \mathrm{~nm}$ pour 4-2Py-SBF, $363 \mathrm{~nm}$ pour 4-3Py-SBF, $365 \mathrm{~nm}$ pour 4-5PmSBF et $369.5 \mathrm{~nm}$ pour 4-4Py-SBF tous décalés vers le rouge en comparaison de l'émission du 4-Ph-SBF (358 nm) (figure 11b). La présence du groupe électrodéficient (au lieu du phényl dans 4-Ph-SBF) induit donc un décalage bathochrome de l'émission dû à la contraction du gap entre la HOMO et la LUMO. Le rendement de fluorescence de 4-2Py-SBF est de 0.17 alors que ceux de 4-3Py-SBF et 44Py-SBF sont proche de celui de 4-Ph-SBF (0.4). L'émission de fluorescence de 4-5Pm-SBF est très faible avec un rendement de 0.04 .

Les comportements électroniques de $4-\mathbf{P O P h}_{\mathbf{2}}-\mathbf{S B F}^{[20]}$ et de son isomère $2-\mathbf{P O P h}_{\mathbf{2}}-\mathbf{S B F}^{[21]}$ sont singuliers dans la série des 4-SBF puisque pour ces deux molécules la rupture de conjugaison entre SBF et son substituant $\mathbf{P O P h}_{\mathbf{2}}$ se fait via l'atome de phosphore. Les spectres d'absorption des deux composés sont similaires avec deux maxima d'absorption à 308 et $322 \mathrm{~nm}$ pour 4-POPh $\mathbf{F}_{2}-\mathbf{S B F}$ et à 308 et $316 \mathrm{~nm}$ pour 2-POPh $\mathbf{2}-\mathbf{S B F}$ quant aux spectres d'émission ils sont tous les deux larges et non

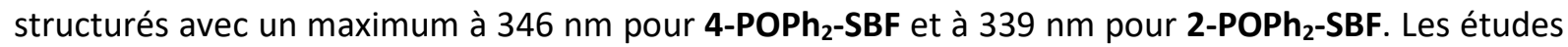
électrochimiques conduisent pour ces deux composés à des HOMO similaires (-6.57 / -6.55 eV pour le 4- et le 2-SBF) et des LUMO différentes (-2.82/-2.73 eV) conduisant à une contraction du gap HOMO-LUMO de 2-POPh ${ }_{2}-\mathbf{S B F}\left(3.82 \mathrm{eV}\right.$ ) au 4-POPh ${ }_{2}-\mathrm{SBF}(3.75 \mathrm{eV})$. Cette contraction du $\Delta \mathrm{E}^{\mathrm{el}}$ est en accord avec celle du $\Delta \mathrm{E}^{\mathrm{opt}}$ signée par le décalage vers le rouge de la bande d'absorption de plus faible énergie (316 nm / $322 \mathrm{~nm})$.

Le spectre d'absorption du 2,7-DiCbz-SBF-4'-POPh ${ }_{2}{ }^{[22]}$ présenté en noir figure 13a, est différent de ceux des 4-SBF présentés précédemment, en effet, c'est un spectre structuré et décalé vers le rouge avec des maxima à 292, 324 et $349 \mathrm{~nm}$ et un $\Delta \mathrm{E}^{\mathrm{opt}}$ mesuré à $3.41 \mathrm{eV}$, similaire à celui de son unité de base 2,7-DiCbz-SBF. Ces bandes d'absorption ont été attribuées aux transitions optiques du fragment le plus conjugué de la molécule (2,7-DiCbz-SBF), le greffage de $\mathrm{POPh}_{2}$ en position 4' n'a donc pas d'effet majeur sur le spectre d'absorption du 2,7-DiCbz-SBF-4'-POPh $\mathbf{P}_{\mathbf{2}}$. Les propriétés électrochimiques conduisent pour 2,7-DiCbz-SBF-4'-POPh 2 à une HOMO à -5.51 eV et une LUMO à -2.10 eV. II est démontré par comparaison avec l'oxydation du 2,7-DiCbz-SBF que la première oxydation se fait sur le fluorène portant les deux unités carbazole. Le $\Delta \mathrm{E}^{\mathrm{el}}$ du 2,7-DiCbz-SBF-4'-POPh ${ }_{2}$ est de $3.41 \mathrm{eV}$ soit 0.1 
eV plus faible que celui du 2,7-DiCbz-SBF montrant une faible influence de la substitution en position 4 du SBF par POPh 2 .
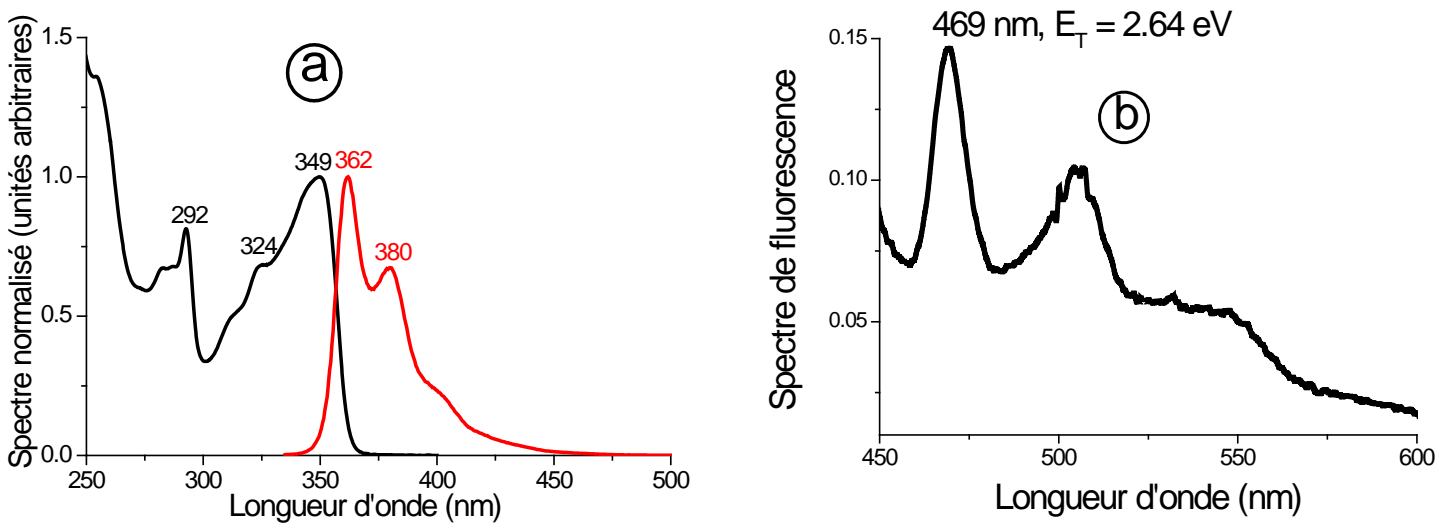

Figure 13. (a) Spectres d'absorption (noir) et d'émission de fluorescence ( $\lambda_{\text {exc }}: 325 \mathrm{~nm}$ ) (rouge) de 2,7-diCbz-SBF-4'$\mathrm{POPh}_{2}$ enregistrés à température ambiante dans le cyclohexane ; (b) Spectre d'émission $\left(\lambda_{\text {exc }}: 315 \mathrm{~nm}\right)$ de 2,7-diCbz-SBF4'-POPh 2 enregistré en matrice gelée de 2-méthyl-THF à $77 \mathrm{~K}$.

Le spectre de fluorescence de 2,7-DiCbz-SBF-4'-POPh 2 est structuré et présente deux maxima à 362 et $380 \mathrm{~nm}$, le rendement de fluorescence est de 0.78 (figure 13a, rouge).

L'enregistrement de l'émission de phosphorescence des 4-SBF à substituant pauvre en électron à basse température conduit à des états triplets compris entre $2.64 \mathrm{eV}$ pour 2,7-DiCbz-SBF-4'-POPh $\mathbf{P}_{2}$ (figure 13b) et $2.79 \mathrm{eV}$ pour 4-3Py-SBF. ${ }^{[12]}$ Comparé au niveau $\mathrm{E}_{\mathrm{T}}$ du SBF (2.87 eV) ou du 4-Ph-SBF (2.77 eV), la substitution par les unités pyridine ou pyrimidine $n^{\prime} i m p a c t e$ que peu $E_{T}$, dont les valeurs restent supérieures à celles du dopant vert $\operatorname{Ir}(\mathrm{ppy})_{3}$ ou du dopant bleu FIrpic. Pour 4-POPh $\mathbf{P}_{2} \mathbf{S B F}, \mathrm{E}_{\mathrm{T}}$ : $2.78 \mathrm{eV}$, la rupture de conjugaison via l'atome de phosphore permet de conserver un $\mathrm{E}_{\mathrm{T}}$ élevé et adapté à une utilisation en matrice hôte pour dopant vert et/ou bleu. En revanche, la valeur du $E_{T} d u$ 2,7-DiCbz-SBF-4'-POPh $(2.64 \mathrm{eV})$ est proche de celle mesurée pour les dérivés 2-SBF et est en accord avec l'extension de conjugaison induite par la substitution d'une unité fluorène en position 2 (et 7), cette valeur de $\mathrm{E}_{\mathrm{T}}$ est trop faible pour envisager I'utilisation de 2,7-DiCbz-SBF-4'-POPh en matrice dans une PhOLED bleue.

En ce qui concerne les propriétés thermiques, la $T_{d}$ des 4-Py-SBF est comprise entre 217 et $242{ }^{\circ} \mathrm{C},{ }^{[12]}$ légèrement plus faible que celle du 4-Ph-SBF $\left(254^{\circ} \mathrm{C}\right) \cdot{ }^{[14]}$ La substitution par une unité pyridine / à une unité phényl conduit donc à une diminution de la stabilité thermique. L'effet inverse est observé pour 4-5Pm-SBF $\left(T_{d}: 277^{\circ} \mathrm{C}^{[13]}\right)$ montrant l'intérêt de la substitution par l'unité pyrimidine sur les propriétés thermiques. L'introduction de l'unité $\mathbf{P O P h _ { 2 }}$ augmente encore cette stabilité avec une $T_{d}$ à $297^{\circ} \mathrm{C}$ pour 4-POPh $-\mathbf{S B F}^{[20]}$ et à $426^{\circ} \mathrm{C}$ pour 2,7-DiCbz-SBF-4'-POPh . $^{[22]}$ La stabilité de cette dernière molécule peut être due à celle de son corps 2,7-DiCbz-SBF pour lequel une $T_{d}$ est reporté à $587^{\circ} \mathrm{C}$. La substitution du second fluorène par l'unité $\mathbf{P O P h}_{2}$ ferait donc chuter la stabilité thermique de plus de $160^{\circ} \mathrm{C}$. 
Les mesures de DSC conduisent à des $\mathrm{T}_{\mathrm{g}}$ comprises entre 81 et $85^{\circ} \mathrm{C}$ pour les dérivés $4-\mathrm{Py}^{\mathrm{SBF}}{ }^{[12]}$ et 4-5Pm-SBF. ${ }^{[13]}$ La substitution par une unité pyridine ou pyrimidine augmente donc la température de transition vitreuse par rapport à la substitution par un phényl $\left(T_{g}: 76^{\circ} \mathrm{C}\right)$. Cette augmentation est la conséquence d'interactions intermoléculaires fortes de type $\mathrm{CH}$.....N. De manière intéressante, 44Py-SBF, 2-4Py-SBF, 4-5Pm-SBF et 2-5Pm-SBF pour lesquels les atomes d'azote sont situés en position externe de la molécule présentent les $T_{g}$ les plus élevées. Enfin, la $T_{g}$ de 2,7-DiCbz-SBF-4'$\mathbf{P O P h}_{2}\left(193^{\circ} \mathrm{C}\right)$ est supérieure à celle des molécules précédentes et à celle de $4-\mathbf{P O P h}_{2}-\mathbf{S B F}\left(127^{\circ} \mathrm{C}\right)$, la substitution des deux unités fluorène du SBF permet d'augmenter sensiblement la $T_{g}$.

Les principales propriétés physicochimiques des six dérivés 4-SBF substitués par un groupement déficitaire en électrons sont résumées dans le tableau 3 avec, pour comparaison, celles de leurs homologues 2-SBF décrits dans la littérature.

Table 3. Propriétés physicochimiques des dérivés 2- et 4-SBF substitués par des groupements déficitaires en électrons.

\begin{tabular}{|c|c|c|c|c|c|c|c|c|}
\hline & $\begin{array}{c}\text { 4-2Py-SBF } \\
{[12]}\end{array}$ & $\begin{array}{c}\text { 4-3Py-SBF } \\
{[12]}\end{array}$ & $\begin{array}{c}\text { 4-4Py-SBF } \\
{[12]}\end{array}$ & $\begin{array}{c}\text { 2-4Py-SBF } \\
{[12]}\end{array}$ & $\begin{array}{c}\text { 4-5Pm-SBF } \\
{[13]}\end{array}$ & $\begin{array}{c}\text { 2-5Pm-SBF } \\
{[13]}\end{array}$ & $\begin{array}{c}\text { 2,7-DiCbz- } \\
\text { SBF-4'-POPh } \\
\text { [22] }\end{array}$ & $\begin{array}{c}\text { 4-POPh } \\
\text { [20] }\end{array}$ \\
\hline$\overline{\lambda_{\max , \text { abs }}(\mathrm{nm})^{\mathrm{a}}}$ & 297,308 & 297,308 & 297,308 & $\begin{array}{c}297,308, \\
321\end{array}$ & $297,308,318$ & $\begin{array}{c}296,308, \\
320\end{array}$ & 292,349 & 308,322 \\
\hline$\Delta \mathrm{E}^{\text {opt a }}$ & 3.80 & 3.81 & 3.75 & 3.70 & 3.72 & 3.66 & 3.41 & 3.75 \\
\hline$\lambda_{\text {max fluo }}(n m)^{a}$ & 370 & 363 & 369.5 & $\begin{array}{l}337,354, \\
370 \text { (sh) }\end{array}$ & 298,311 & 338,355 & 362,380 & 346 \\
\hline$\lambda_{\text {max } \text { fluo, }}$ thin film $(n m)^{b}$ & 366 & 365 & 377 & 375,425 & 365 & 355,366 & - & 353 \\
\hline$\lambda_{\text {max } \text { phosphor }}(77 \mathrm{~K})(\mathrm{nm})$ & $448^{d}$ & $444^{d}$ & $451^{d}$ & $480^{d}$ & $372^{\mathrm{e}}$ & $480^{e}$ & $469^{d}$ & - \\
\hline $\mathrm{E}_{\mathrm{T}}(\mathrm{eV})$ & $2.76^{d}$ & $2.79^{d}$ & $2.74^{d}$ & $2.58^{d}$ & $2.75^{\mathrm{e}}$ & $2.58^{\mathrm{e}}$ & $2.64^{d}$ & 2.78 \\
\hline$\Phi_{\text {sol }}{ }^{c}$ & 0.17 & 0.40 & 0.40 & 0.55 & 0.04 & 0.02 & 0.87 & - \\
\hline $\begin{array}{l}\text { Durée de vie de } \\
\text { fluorescence }(\mathrm{ns})^{\text {a }}\end{array}$ & 3.88 & 3.89 & 3.80 & 1.03 & 0.61 & 0.44 & 4.6 & - \\
\hline $\begin{array}{l}\text { Constante Radiative } \\
\left(\mathrm{k}_{\mathrm{r}}\right)\left(10^{8} \mathrm{~s}\right)\end{array}$ & 0.44 & 1.03 & 1.05 & 5.34 & 0.66 & 0.46 & 6.4 & - \\
\hline $\begin{array}{l}\text { Constante non-radiative } \\
\left(\mathrm{k}_{\mathrm{nr}}\right)\left(10^{8} \mathrm{~s}\right)\end{array}$ & 2.14 & 1.54 & 1.58 & 4.37 & 16 & 22 & 1.8 & - \\
\hline $\mathrm{HOMO}^{f}$ & -5.88 & -5.90 & -5.88 & -5.78 & -5.97 & -5.96 & -5.51 & -6.57 \\
\hline LUMO $^{f}$ & -2.12 & -2.01 & -2.11 & -2.26 & -2.23 & -2.30 & -2.10 & -2.82 \\
\hline$T_{d}$ & 242 & 220 & 217 & 181 & 277 & 242 & 426 & 297 \\
\hline$T_{g}$ & 81 & 81 & 84 & 92 & 85 & 94 & 193 & 127 \\
\hline
\end{tabular}

${ }^{a}$ dans le cyclohexane ${ }^{b}$ à partir d'une solution à $10 \mathrm{~g} / \mathrm{L}^{\mathrm{c}}$ référence sulfate de quinine dans $\mathrm{H}_{2} \mathrm{SO}_{4} 1 \mathrm{~N}$, dans une matrice gelée de 2 -Me-THF à $77 \mathrm{~K}$, e dans une matrice gelée de méthylcyclohexane/2-méthylpentane mixture (1:1) à $77 \mathrm{~K}$, ${ }^{\mathrm{f}}$ à partir des potentiels de seuil en voltammétrie cyclique.

La plupart de ces composes ont été utilisés comme matrice hôte en PhOLEDs pour des diodes vertes ou bleues. Les performances des diodes à base de Py-SBF ou Pm-SBF, présentées dans la tableau 4, peuvent être directement comparées à celles utilisant 4-Ph-SBF comme matériaux hôte car tous les dispositifs ont la même architecture: ITO/CuPc(10 nm)/NPB(40 nm)/TCTA(10 nm)/matrice hôte:dopant(20 nm)/TPBi(40 nm)/LiF(1.2 nm)/Al(100 nm). Le rendement des diodes vertes à base de pyridine ou de pyrimidine est supérieur à celui des diodes à base de SBF ou 4-Ph-SBF, montrant l'intérêt du groupement électrodéficient en position 4 du SBF. On notera principalement une augmentation de I'EQE atteignant 15.7 \% avec 4-4Py-SBF comme matrice hôte. ${ }^{[12]}$ 
Tableau 4. Performances de diodes vertes ou bleues utilisant, comme matrice hôte, des dérivés de 4-SBF électrodéficients.

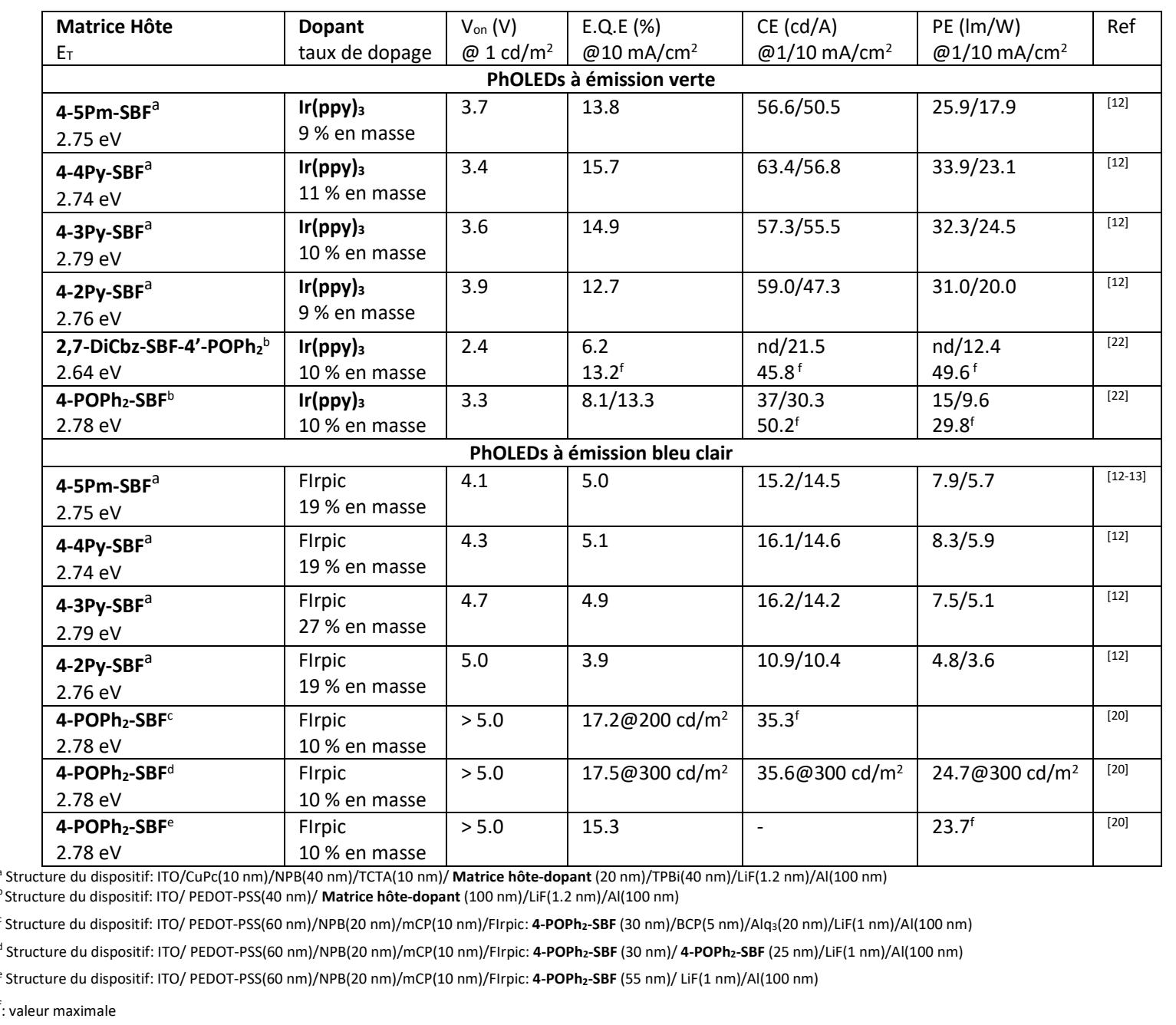

Le composé bipolaire 2,7-DiCbz-SBF-4'-POPh ${ }_{2}$ a été utilisé comme matrice hôte dans un dispositif simplifié de type monocouche (sans couche injectrice et/ou bloqueuse de charge). La simplification de l'architecture des dispositifs est importante dans le but de simplifier la préparation des dispositifs et d'en diminuer le coût. Avec une telle architecture, la tension de fonctionnement de la diode est très faible, $2.4 \mathrm{~V}$, et un EQE de $13.2 \%$ a été atteint. Avec une architecture similaire, une diode utilisant 4-POPh${ }_{2}$-SBF comme matrice hôte a une tension de fonctionnement de $3.3 \mathrm{~V}$ (plus élevée de presque $1 \mathrm{~V}$ ) et atteint un EQE de $\mathbf{1 3 . 3} \%$. Ceci montre que le caractère bipolaire de 2,7-DiCbz-SBF-4'$\mathbf{P O P h}_{2}$ permet de réduire la tension de seuil, mais que l'efficacité du transfert énergétique matrice hôte vers dopant est principalement due à la substitution du SBF en position 4 par le groupe $\mathbf{P O P h}_{\mathbf{2}}$.

Excepté les PhOLEDs utilisant 4-2Py-SBF comme matrice hôte pour le dopant Flrpic qui ont un EQE à 3.9 \%, les autres dispositifs utilisant un dérivé de 4-Py-SBF ou le 4-5Pm-SBF comme matrice hôte ont des performances comparables avec un EQE proche de $5 \%$ et une tension de fonctionnement proche de $4 \mathrm{~V}$. Ces performances montrent l'intérêt de la substitution en position 4 du SBF par un 
groupement déficitaire en électron. Les diodes à matrice-hôte 4-POPh $\mathbf{P}_{2}-\mathbf{S B F}$ ont des architectures différentes et présentent des performances plus élevées. Dans une diode utilisant comme matrice hôte et comme couche de transport d'électron, un rendement de 17.5 \% (CE : 35.5 cd/A et PE 24.7 $\mathrm{Im} / \mathrm{W}$ ) est atteint. En présence d'une couche de transport d'électron BCP, les performances sont du même ordre de grandeur (EQE : 17.2 \% et $\mathrm{CE}: 35.3 \mathrm{~cd} / \mathrm{A}$ ) alors que dans un dispositif sans couche de transporteur d'électron, les performances sont plus faibles (EQE : $15.9 \%$ et PE $2.7 \mathrm{Im} / \mathrm{W}$ ). Tous ces résultats mettent en évidence l'efficacité du 4-POPh $2-S B F$ comme matrice hôte pour le dopant Flrpic.

\section{4-SBF à substituant riche en électrons}

La littérature ne présente que cinq exemples de dérivés du SBF substitué en position 4 par un groupement donneur d'électrons utilisés comme matériau hôte en PhOLEDs, ils sont reportés sur la figure 14. Ces molécules sont dérivées du SBF substitué en C4 par un dibenzofurane (4-DBF-SBF) ${ }^{[23]}$, dibenzothiophène (4-DBT-SBF), ${ }^{[24]}$ spiro-phenylacridine-fluorene (4-SFA-SBF), ${ }^{[25]} \mathrm{N}$-phenylcarbazole (4-PhCBz-SBF) ${ }^{[26]}$ ou trimethoxyphényl (4-PhOMe 3 -SBF). ${ }^{[26]}$

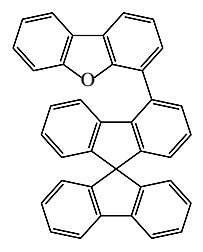

4-DBF-SBF

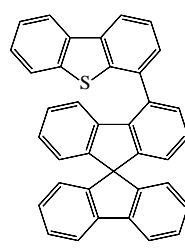

4-DBT-SBF

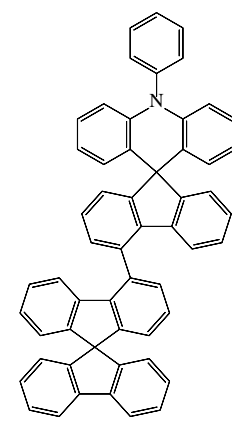

4-SFA-SBF

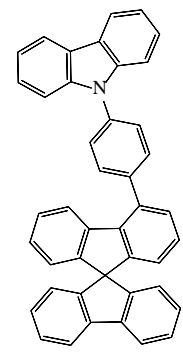

4-PhCBz-SBF

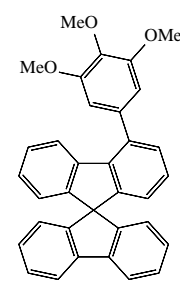

4-PhOMe 3 -SBF

Figure 14. Composés dérivés du 4-SBF substitués par un groupement riche en électrons.

Ces cinq molécules ont été synthétisées par couplage de Suzuki entre 4-Br-SBF et le dérivé boronique adapté à l'obtention de la molécule finale avec des rendements compris entre 52 et $83 \%$. Seuls 4PhCBz-SBF et 4-PhOMe $\mathrm{OMBF}_{3} \mathrm{SBF}$ ont pu être cristallisés permettant d'accéder à leurs structures RX. ${ }^{[26]} \mathrm{De}$ ces structures, un angle de $45.4^{\circ}$ (4-PhCBz-SBF) et de $78^{\circ}$ (4-PhOMe 3 -SBF) est mesuré entre le phényl et l'unité fluorène à laquelle il est lié. Cet angle est donc un peu plus faible dans 4-PhCBz-SBF et un peu plus fort dans 4-PhOMe 3 -SBF que dans 4-Ph-SBF $\left(>51.2^{\circ}\right)$. Ceci montre que non seulement l'encombrement du substituant du SBF (phényl, phénylcarbazole ou triméthoxyphényl) mais aussi la substitution du groupe phényl lui-même (substitué en para dans 4-PhCBz-SBF et en méta et para dans 4-PhOMe $-\mathrm{SBF}$ ) a un impact sur l'angle entre SBF et son substituant et donc sur l'intensité de la rupture de conjugaison entre le SBF et son substituant en position 4. 
Les principales caractéristiques physicochimiques des cinq dérivés 4-SBF substitués par un groupement riche en électrons sont résumées dans le tableau 5. Hormis celui du 4-PhCBz-SBF, les spectres d'absorption des quatre autres composés sont structurés avec pour tous un maximum à 309 $\mathrm{nm}$ et une queue de bande plus ou moins intense entre 309 et $350 \mathrm{~nm}$ signant une faible conjugaison entre le fluorène et le substituant. Cette conjugaison peu intense conduit à des $\Delta \mathrm{E}_{\mathrm{opt}}$ compris entre 3.7 et $3.93 \mathrm{eV}$ (3.7 eV pour 4-DBT-SBF, 3.82 et $3.83 \mathrm{eV}$ pour 4-PhOMe - -SBF et 4-DSF-SBF et $3.93 \mathrm{eV}$ pour 4-SFA-SBF), proches de celui de 4-Ph-SBF (3.82 eV). Le spectre du 4-PhCBz-SBF est lui aussi structuré avec des maxi à 293, 309 nm auxquels d'ajoutent deux bandes centrées à 325 et $340 \mathrm{~nm}$ signatures de transitions impliquant le fragment $\mathrm{N}$-phénylcarbazole.

Tableau 5. Propriétés physicochimiques des 4-DBF-SBF ${ }^{[23]}$ 4-DBT-SBF, ${ }^{[24]}$ 4-PhOMe3-SBF, ${ }^{[26]}$ 4-PhCBz-SBF, ${ }^{[26]}$ et 4-SFA-SBF ${ }^{[25]}$

\begin{tabular}{|c|c|c|c|c|c|}
\hline & $\begin{array}{c}\text { 4-DBF-SBF } \\
{[23]}\end{array}$ & $\begin{array}{c}\text { 4-DBT-SBF } \\
{[24]}\end{array}$ & $\underset{[26]}{\text { 4-PhOMes-SBF }}$ & $\begin{array}{c}\text { 4- PhCBz-SBF } \\
{[26]}\end{array}$ & $\begin{array}{c}\text { 4-SFA-SBF } \\
{[25]}\end{array}$ \\
\hline$\lambda_{\max , \text { abs }}(\mathrm{nm})$ & $279,309^{a}$ & $\begin{array}{c}286, \\
309,329^{a}\end{array}$ & $297,309^{b}$ & $\begin{array}{c}293,309 \\
325 \text { (ep.), } 340^{\text {b }}\end{array}$ & $308^{a}$ \\
\hline$\Delta \mathrm{E}_{\mathrm{opt}}(\mathrm{eV})^{\mathrm{e}}$ & 3.79 & 3.70 & 3.82 & 3.58 & $\begin{array}{c}3.93 \\
\text { Je mettrai } \\
\text { bien } 3.61 \mathrm{eV}\end{array}$ \\
\hline$\lambda_{\max , \text { fluo }}(\mathrm{nm})$ & $369^{a}$ & $355^{\mathrm{a}}$ & $375^{b}$ & $345 / 361^{b}$ & $380^{c}$ \\
\hline$\lambda_{\max , \text { phospho }}(77 \mathrm{~K})(\mathrm{nm})$ & $440^{d}$ & $441^{d}$ & $437^{d}$ & $442^{d}$ & $437^{d}$ \\
\hline $\mathrm{E}_{\mathrm{T}}(\mathrm{eV})^{\mathrm{f}}$ & 2.82 & 2.82 & 2.84 & 2.81 & 2.83 \\
\hline $\mathrm{T}_{\mathrm{g}}\left({ }^{\circ} \mathrm{C}\right)$ & 124 & 131 & 90 & 127 & 188 \\
\hline $\mathrm{T}_{\mathrm{d}}\left({ }^{\circ} \mathrm{C}\right)$ & 305 & 318 & 268 & 311 & 402 \\
\hline HOMO (eV) & $-6.00^{a, g}$ & $-6.3^{h}$ & $-5.62^{a, g}$ & $-5.52^{a, g}$ & $-5.33^{a, l}$ \\
\hline LUMO (eV) & $-2.17^{a, j}$ & $-2.7^{i}$ & $-1.91^{\mathrm{a}, \mathrm{g}}$ & $-1.97^{a, g}$ & $\begin{array}{c}-1.38^{i} \\
\text { Je mettrai } \\
\text { bien }-1.72 \\
\text { eV }\end{array}$ \\
\hline$\Delta \mathrm{E}^{\mathrm{el}}(\mathrm{eV})$ & $3.83^{k}$ & - & $3.71^{k}$ & $3.55^{k}$ & - \\
\hline
\end{tabular}

${ }^{a}$ dans $\mathrm{CH}_{2} \mathrm{Cl}_{2}$; ${ }^{\mathrm{b}}$ dans le cyclohexane ; ${ }^{\mathrm{c}}$ dans le toluène; ${ }^{\mathrm{d}}$ une matrice gelée de 2-Me-THF à $77 \mathrm{~K}$; ${ }^{\mathrm{e}} \Delta E^{\text {opt }}$ calculé à partir de la longueur d'onde de seuil d'absorption $\left(1239.84 / \lambda_{\text {seuil }}(\mathrm{nm})\right) ;{ }^{\mathrm{f}}$ calculé à partir du premier pic de phosphorescence $\left(1239.84 / \lambda_{\text {seuil }}(\mathrm{nm})\right) ;{ }^{\mathrm{g}}$ à partir des potentiels de seuil en voltammétrie cyclique ; ${ }^{h}$. à partir de spectrométrie photoélectronique UV ; ${ }^{i} L U M O=\Delta E^{o p t}-H O M O ;{ }^{j}$ dans le $D M F$, $k$ $\Delta E^{\mathrm{el}}=\mathrm{HOMO}-\mathrm{LUMO} ;{ }^{\prime}$ à partir du potentiel de demi-vague en voltammétrie cyclique

Concernant les spectres de fluorescence, excepté celui du 4-PhCz-SBF, ils sont tous non structurés avec un maximum centré entre $355 \mathrm{~nm}$ pour 4-DBT-SBF et $380 \mathrm{~nm}$ pour 4-SFA-SBF (369 nm pour 4DBF-SBF et $375 \mathrm{~nm}$ pour 4-PhOMe - -SBF) similaire à celui du 4-Ph-SBF centré à $358 \mathrm{~nm}$. Le spectre de fluorescence de 4-PhCz-SBF est structuré avec deux maxima à 345 et $361 \mathrm{~nm}$ et un épaulement autour de $380 \mathrm{~nm}$. Cette émission, similaire à celle du N-PhCz (341, 356 et 375(sh) nm)) et apparaissant dans la zone d'émission du 4-Ph-SBF est différente des émissions des dérivés 4-SBF présentées précédemment. Le spectre bien résolu et le faible déplacement de Stokes $(5 \mathrm{~nm})$ peut être expliqué par la rigidité du phényl-carbazole qui n'autorise que peu de réarrangements structuraux entre l'état excité et l'état fondamental contrairement à ce qui est observé pour les autres 4-SBF. 
Les états triplets des molécules sont tous proches de $2.82 \mathrm{eV}$, ces molécules sont donc toutes aptes à être utilisées comme matrice hôtes pour le dopant bleu Flrpic.

Les $\mathrm{T}_{\mathrm{g}}$ des molécules augmentent de $90{ }^{\circ} \mathrm{C}$ pour 4-PhOMe 3 -SBF à $188^{\circ} \mathrm{C}$ pour 4-SFA-SBF, (4-DBF-SBF, 4-DBT-SBF et 4-PhCBz-SBF ont des $\mathrm{T}_{\mathrm{g}}$ proche de $127^{\circ} \mathrm{C}$ ) et sont toutes plus élevées que celle du 4-PhSBF $\left(76^{\circ} \mathrm{C}\right)$. Cette variation est en accord avec une augmentation de la $\mathrm{Tg}$ avec la dimension des molécules telle que celle observée avec les oligomères de SBF.

Les $\mathrm{T}_{\mathrm{d}}$ augmentent de $268^{\circ} \mathrm{C}$ pour 4-PhOMe 3 -SBF à $402{ }^{\circ} \mathrm{C}$ pour 4-SFA-SBF (4-DBF-SBF, 4-DBT-SBF et 4-PhCBz-SBF ont des $T_{d}$ proches de $311^{\circ} \mathrm{C}$ ) en suivant la même tendance que celle de la $T_{\mathrm{g}}$. Toutes ces molécules ont donc une haute stabilité thermique, facteur majeur pour la stabilité des dispositifs

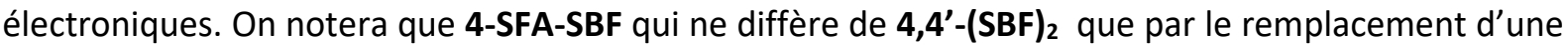
unité fluorène par une unité phénylacridine présente des $T_{g} / T_{d}\left(188 / 402{ }^{\circ} \mathrm{C}\right)$ plus élevées que celles de $\mathbf{4 , 4}$ '-(SBF) ${ }_{2}\left(170 / 360^{\circ} \mathrm{C}\right)$ montrant l'intérêt de l'unité phénylacridine sur la stabilité thermique de molécules à propriétés électroniques similaires $\left(\lambda_{\mathrm{PL}}(\mathrm{nm}) / \mathrm{E}_{\mathrm{T}}(\mathrm{eV}): 373 / 2.81\right.$ pour $\mathbf{4 , 4} \mathbf{4}^{\prime}-(\mathrm{SBF})_{2}$ et $380 / 2.83$ pour 4-SFA-SBF).

Table 6. Performances de diodes vertes ou bleues utilisant, comme matrice hôte, des dérivés de 4-SBF à substituant riche en électrons

\begin{tabular}{|c|c|c|c|c|c|c|}
\hline $\begin{array}{l}\text { Matrice Hôte } \\
E_{T}\end{array}$ & $\begin{array}{l}\text { Dopant } \\
\text { doping level }\end{array}$ & $V_{\text {on }}(V)$ & $\begin{array}{l}\text { E.Q.E (\%) } \\
\text { (max value) }\end{array}$ & $\begin{array}{l}\text { CE (cd/A) } \\
\text { (max value) }\end{array}$ & $\begin{array}{l}\text { PE (Im/W) } \\
\text { (max value) }\end{array}$ & Ref \\
\hline \multicolumn{7}{|c|}{ PhOLEDs à émission verte } \\
\hline $\begin{array}{l}\text { 4-DBF-SBF } \\
2.82 \mathrm{eV}\end{array}$ & $\begin{array}{l}\operatorname{Ir}(\text { ppy })_{2} \text { acac } \\
8 \text { wt \% }\end{array}$ & 4.9 & 21.2 & 64.4 & 49.7 & \begin{tabular}{l|l}
$23]$ \\
\end{tabular} \\
\hline $\begin{array}{l}\text { 4-DBT-SBF } \\
2.82 \mathrm{eV}\end{array}$ & $\begin{array}{l}\operatorname{Ir}\left(\text { ppy) }{ }_{2 a c a c}\right. \\
8 \text { wt \% }\end{array}$ & 5.3 & 16.3 & 62.0 & 45.9 & 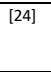 \\
\hline $\begin{array}{l}\text { 4-PhOMe }-\mathrm{SBF}^{\mathrm{c}} \\
2.84 \mathrm{eV}\end{array}$ & $\begin{array}{l}\operatorname{Ir}(\text { ppy })_{3} \\
10 \text { wt \% }\end{array}$ & 4.15 & 20.2 & 78 & 48.1 & [26] \\
\hline $\begin{array}{l}\text { 4-PhCBz-SBF } \\
2.81 \mathrm{eV}\end{array}$ & $\begin{array}{l}\operatorname{Ir}(\text { ppy })_{3} \\
10 \text { wt } \%\end{array}$ & 3.7 & 17.5 & 67.9 & 45.4 & [26] \\
\hline \multicolumn{7}{|c|}{ PhOLEDs à émission bleu clair } \\
\hline $\begin{array}{l}\text { 4-DBF-SBF } \\
2.82 \mathrm{eV}\end{array}$ & $\begin{array}{l}\text { FIrpic } \\
8 \text { wt \% }\end{array}$ & 5.6 & 7.5 & 22.2 & 16.4 & [23] \\
\hline $\begin{array}{l}\text { 4-DBT-SBF } \\
2.82 \mathrm{eV}\end{array}$ & $\begin{array}{l}\text { FIrpic } \\
8 \text { wt \% }\end{array}$ & 6.7 & 10.3 & 23.5 & 16.6 & [24] \\
\hline $\begin{array}{l}\text { 4-PhOMe }-\mathrm{SBF}^{\mathrm{c}} \\
2.84 \mathrm{eV}\end{array}$ & $\begin{array}{l}\text { FIrpic } \\
20 \text { wt \% }\end{array}$ & 4.0 & 9.6 & 24.2 & 13.9 & [26] \\
\hline $\begin{array}{l}\text { 4-PhCBz-SBF } \\
2.81 \mathrm{eV}\end{array}$ & $\begin{array}{l}\text { FIrpic } \\
17 \text { wt \% }\end{array}$ & 3.75 & 6.7 & 18 & 11 & {$[26]$} \\
\hline $\begin{array}{l}\text { 4-SFA-SBF } \\
2.83 \mathrm{eV}\end{array}$ & $\begin{array}{l}\text { Flrpic } \\
10 \text { wt \% }\end{array}$ & 2.9 & 19.1 & 41 & 42 & [25] \\
\hline
\end{tabular}

a Architecture du dispositif: ITO/MoO $3(10 \mathrm{~nm}) / \mathrm{NPB}(35 \mathrm{~nm}) / \mathrm{TCTA}(10 \mathrm{~nm}) /$ Matrice hôte-dopant(15 nm)/TPBi(65 nm)/Liq(20 nm)/Al(100 nm) ${ }^{\mathrm{b}}$ Architecture du dispositif: ITO/MoO $3(10 \mathrm{~nm}) / \mathrm{NPB}(80 \mathrm{~nm}) / \mathrm{TCTA}(5 \mathrm{~nm}) /$ Matrice hôte-dopant(20 nm)/TPBi(40 nm)/Liq(2 nm)/Al(100 nm)

${ }^{c}$ Architecture du dispositif: ITO/CuPc(10 nm)/NPB(40 nm)/TCTA(10 nm)/Matrice hôte-dopant(20 nm)/TPBi(40 nm)/LiF(1.2 nm)/AI(100 nm)

${ }^{d}$ Architecture du dispositif: ITO/ $\mathrm{MoO}_{3}(10 \mathrm{~nm}) / \operatorname{TAPC}(60 \mathrm{~nm}) /$ Matrice hôte-dopant $(15 \mathrm{~nm}) / \operatorname{TmPyPB}(35 \mathrm{~nm}) / \mathrm{LiF}(1 \mathrm{~nm}) / \mathrm{Al}(120 \mathrm{~nm})$

Les cinq composés ont été utilisés comme matrice hôte dans des PhOLEDs vertes et/ou bleues. Concernant les diodes vertes, les dopants ne sont pas les mêmes pour les diodes à matrice hôte 4DBF-SBF ou 4-DBT-SBF qui utilisent le dopant $\operatorname{Ir}(\text { ppy })_{2}$ acac $\left(\mathrm{E}_{\mathrm{T}}: 2.40 \mathrm{eV}\right)$ dans une architecture similaire. Des performances de ces diodes on notera que 4-DBF-SBF est une matrice hôte plus efficace que 4-DBT-SBF et conduit à un EQE supérieur à $20 \%$. La tendance inverse est observée pour 
lorsque ces matrices sont utilisées avec le dopant bleu FIrpic, montrant la difficulté à rationaliser l'effet des matrices sur l'efficacité du transfert énergétique vers le dopant.

Les diodes à base de 4-PhOMe 3 -SBF ou 4-PhCBz-SBF qui utilisent le dopant $\operatorname{Ir}(\mathrm{ppy})_{3}$ ou le dopant FIrpic dans une architecture de diode similaire, montrent elles que les meilleures performances sont obtenues avec la matrice 4-PhOMe 3 -SBF quelle que soit la couleur émise, par contre la tension de fonctionnement des diodes est plus basse avec 4-PhCBz-SBF comme dopant.

Les performances des diodes utilisant 4-SFA-SBF comma matrice hôte pour le dopant bleu FIrpic sont supérieures à toutes les diodes bleues obtenues en terme de faible tension de seuil (2.9 V) et d'EQE (19.1 \%), l'architecture de diode utilisée étant différente des précédentes, il est malheureusement difficile de conclure sur l'origine de ces bonnes performances (matrice ou architecture ?). Ces résultats montrent tout de même l'intérêt de la substitution du SBF en C4 par la phénylacridine pour améliorer les performances des PhOLEDs bleues.

\section{Autres 4-SBF utilisé en électronique organique}

Les 3 chapitres précédents ont présenté 17 dérivés du SBF substitués en position 4 par un groupement aryle hydrocarboné (partie 2), un groupement déficitaire en électron (partie 3) ou un groupement riche en électrons (partie 4), leurs propriétés physicochimiques et les performances des PhOLEDs utilisant ces molécules comme matrice pour les dopants phosphorescent vert ou bleu. Cette partie 5 réunit huit molécules $4-\mathrm{SBF}$, substituées par différents groupements dont les applications ne visent pas spécifiquement une utilisation comme matrice hôte dans les PhOLEDs bleues. Ces molécules sont divisée en deux catégories, (i) celles dont le substituant en position 4 peut donner lieu à un encombrement stérique qui peut donc induire une rupture de conjugaison entre SBF et le substituant (molécules 4-T-DKPP-SBF ${ }_{2}{ }^{[27]}$ 4-AP-SBF ${ }^{[28]}$ et DSF-AP ${ }^{[29]}$ en haut de la figure 15 ) et (ii) celles dont le substituant est peu encombré (acétylène ou vinyl) (4-EPHDB-SBF, ${ }^{[30]}$ 4-EPHDBA$\mathrm{SBF}^{\left[{ }^{[30]}\right.}{ }^{4-V T P A-S B F},{ }^{[31]} 4-\mathrm{VCbz}-\mathrm{SBF},{ }^{[31]} 4-\mathrm{VTPA}-\mathrm{SBF}(\boldsymbol{t}-\mathrm{Bu})_{2}{ }^{[32]}$ en bas de la figure $\left.{ }^{15}\right)$.

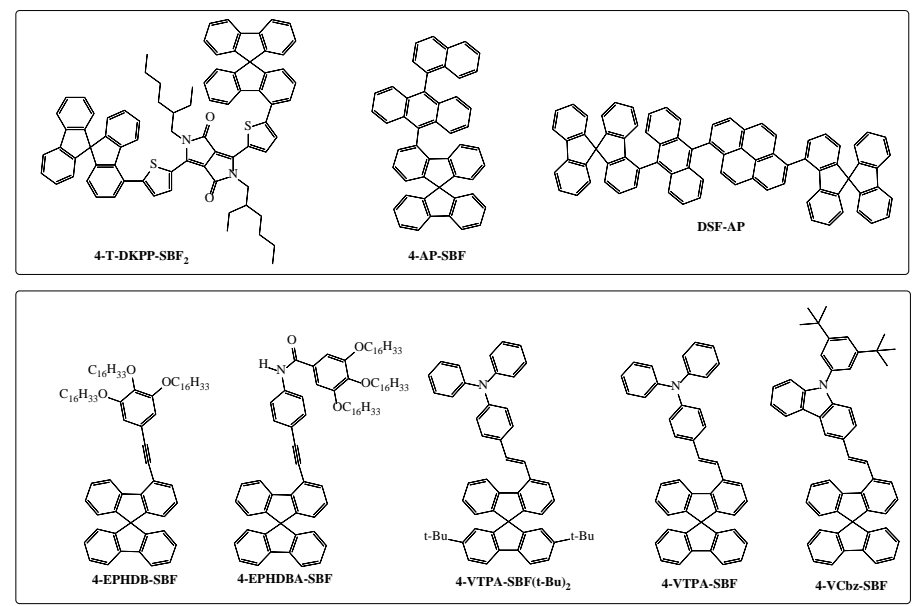

Figure 15. Autres dérivés du 4-SBF 
Sans rentrer dans le détail des propriétés, le tableau suivant résume les principales caractéristiques physicochimiques des huit molécules qui ont été utilisées comme émetteur fluorescents bleus : 4EPHDB-SBF, ${ }^{[30]}$ 4-EPHDBA-SBF, ${ }^{[30]}$ comme couche émissive en OLED fluorescente : DSF-AP, ${ }^{[29]}$ comme dopant fluorescent de la matrice organique MADN (2-méthyl-9,10-di(2-naphtyl)anthracène) : 4VTPA-SBF, ${ }^{[31]}$ 4-VCbz-SBF, ${ }^{[31]}$ et $\left.{ }^{4-V T P A-S B F(t-B u}\right)_{2},{ }^{[32]}$ comme matrice hôte pour le dopant

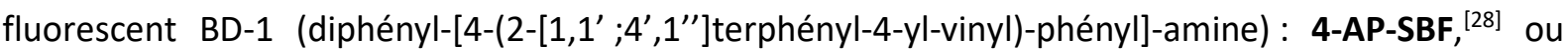
comme transporteur de trous pour des cellules solaires : 4-T-DKPP-SBF ${ }_{2}{ }^{[27]}$

Tableau 8. Propriétés physicochimiques des 4-TDKPP-SBF 2 , 4-AP-SBF, ${ }^{[28]}$ DSF-AP, ${ }^{[29]}$ 4-EPHDBA-SBF, 4-EPHDBSBF, 4-VTPA(t-Bu) $2-S B F, 4-V T P A-S B F^{[32]}$ et 4-VCbz-SBF ${ }^{[31]}$

\begin{tabular}{|c|c|c|c|c|c|c|c|c|}
\hline & $\underset{[27]}{\text { 4-TDKPP-SBF }}{ }_{2}$ & $\begin{array}{c}\text { 4-AP-SBF } \\
{[28]}\end{array}$ & $\begin{array}{c}\text { DSF-AP } \\
{[29]}\end{array}$ & $\begin{array}{c}\text { 4-EPHDBA-SBF } \\
{[30]}\end{array}$ & $\begin{array}{c}\text { 4-EPHDB-SBF } \\
\text { [30] }\end{array}$ & $\begin{array}{c}\text { 4-VTPA-SBF } \\
{[31]}\end{array}$ & $\underset{[31]}{\text { 4-VCbz-SBF }}$ & 4-VTPA-SBF(t-Bu) ${ }_{2}$ \\
\hline$\lambda_{\max , \text { abs }}(\mathrm{nm})$ & $530,570^{a}$ & 394 & $351,379,400^{a}$ & $309,326,348^{h}$ & $309,319,341$ & $371^{i}$ & $338^{i}$ & $370^{i}$ \\
\hline$\Delta \mathrm{E}_{\text {opt }}(\mathrm{eV})$ & $2.02^{\mathrm{b}}$ & $3.0^{\mathrm{b}}$ & $2.94^{\mathrm{b}, \mathrm{g}}$ & $3.45^{\mathrm{b}}$ & $3.49^{b}$ & $2.91^{\mathrm{j}}$ & $3.13^{j}$ & $2.93^{\mathrm{b}, \mathrm{g}}$ \\
\hline$\lambda_{\text {max,fluo }}(\mathrm{nm})$ & & $403 / 413$ & $443^{a}$ & $371^{\mathrm{h}}$ & $403^{h}$ & $470^{i}$ & $430^{i}$ & $468^{i}$ \\
\hline $\mathrm{T}_{\mathrm{g}}\left({ }^{\circ} \mathrm{C}\right)$ & & $>170$ & & & & & & \\
\hline $\mathrm{T}_{\mathrm{d}}\left({ }^{\circ} \mathrm{C}\right)$ & & & 351 & & & & & \\
\hline HOMO (eV) & $-5.65^{c}$ & & $-5.78^{e}$ & & & $-5.57^{e}$ & $-5.63^{e}$ & $-5.59^{e}$ \\
\hline LUMO (eV) & $-3.75^{c}$ & & $-2.84^{f}$ & & & $-2.66^{f}$ & $-2.5^{f}$ & $-2.66^{f}$ \\
\hline$\Delta \mathrm{E}^{\mathrm{el}}(\mathrm{eV})$ & $1.90^{d}$ & - & & & & & & \\
\hline
\end{tabular}

${ }^{\mathrm{a}}$ dans le chloroforme $;{ }^{\mathrm{b}} \Delta E^{\text {opt }}$ calculé à partir de la longueur d'onde de seuil d'absorption $\left(1239.84 / \lambda_{\text {seuil }}(\mathrm{nm})\right) ;{ }^{\mathrm{c}}$ à partir des potentiels de seuil en voltammétrie cyclique dans $\mathrm{CH}_{2} \mathrm{Cl}_{2}+{ }^{+}$

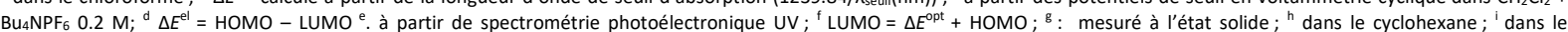
dichlorométhane ; obtenu par l'intersection entre le spectre d'absorption et le spectre de photoluminescence

\section{Perspectives et évolution}

Cette revue fait le point sur une nouvelle famille de molécule dérivées du SBF, molécule centrale de l'électronique organique, substituées, non pas position para (comme classiquement sur le C2) mais en position ortho (sur le C4). Cette position de substitution peut entrainer une gêne stérique entre le fluorène et son substituant qui se manifeste par un angle important entre les deux unités et une rupture de conjugaison entre le SBF et son substituant. Des exemples rapportés ci-dessus, on peut voir que la rupture de conjugaison peut être totale ou partielle et que cette modulation de la force de la rupture est une voie d'accès intéressante à la modulation des propriétés électroniques des 4SBF. La substitution en position 4 par des groupements riche ou pauvre en électron permet aussi de moduler les propriétés des 4-SBF par ajustement des niveaux HOMO ou LUMO. II est aussi mis en évidence que la stabilité thermique des 4-SBF augmente avec la dimension du substituant avec des molécules à très haute température de décomposition ().

Concernant les applications de cette nouvelle famille de molécules comme matrice hôte pour les dopants phosphorescents, la rupture de conjugaison entre le SBF et son substituant permet à la molécule 4-SBF de conserver un large écart HOMO/LUMO et un état triplet élevé, permettant à ces molécules d'être adaptées au dopant bleu. Des PhOLEDs à rendements quantiques externes (EQE) proche de $20 \%$ ont été obtenues (EQE : 22\% avec la matrice hôte 4,3'-(SBF) ; $_{2} ; 17.2 \%$ avec 4-POPh $\mathbf{2}^{-}$ SBF et $20.2 \%$ avec 4-Ph(OMe) $\left.)_{3}-\mathrm{SBF}\right)$, ces EQE sont dans les plus hauts reportés à ce jour pour des 
PhOLEDs bleues, ce qui montre l'intérêt de l'architecture moléculaire 4-SBF. On rappellera qu'une diode à matrice hôte SBF ne conduit qu'à un EQE de $8.5 \%$.

Les perspectives sont donc nombreuses pour les dérivés 4-SBF et la plus simple à envisager est l'accès à un matériaux bipolaire possédant un groupement pauvre en électron sur le carbone $\mathrm{C} 4$ et un groupement riche en électrons sur le carbone en C4' du SBF permettant de conserver un haut état triplet et un large écart HOMO/LUMO tout en facilitant l'injection et le transport des charges dans la matrice.

\section{Remerciements:}

Cyril Poriel et Joëlle Rault-Berthelot souhaitent remercier l'Université de Rennes 1, le CNRS, le Ministère de la Recherche, l'Agence Nationale de la Recherche, l'ADEME et la Région Bretagne pour leurs soutiens financiers. Les auteurs souhaitent également remercier l'ensemble des collaborateurs qui ont participé à ce travail et tout particulièrement les Drs. M Romain, $\mathrm{S}$. Thiery et $\mathrm{C}$. Quinton pour la synthèse et l'analyse physico-chimique des molécules issues de notre groupe présentées dans cet article. Nos remerciements vont aussi à B. Geffroy et au Dr. Denis Tondelier et leurs collaborateurs qui ont fabriqué et testé les PhOLED vertes et bleus utilisant ces molécules comme matériaux hôtes. 


\section{Acronymes: (à rechecker et mettre en français si necessaire)}

2-Br-SBF: 2-bromo-9,9'-spirobifluorene

2,2-Br2-SBF: 9,9'-Spirobi[9H-fluorene], 2,7-dibromo-

2-DBF-SBF: 4-(9,9'-spirobi[9H-fluoren]-2-yl)-dibenzofuran

2-DBT-SBF: 4-(9,9'-spirobi[9H-fluoren]-2-yl)-dibenzothiophene

2,7-DiCbz-SBF-4'-POPh ${ }_{2}$ : 9,9'-(4'-diphenyl-phosphine oxide-9,9'-spirobi[9H-fluorene]-2,7-diyl)bis-9H-Carbazole

2-Ph-SBF: 2-phenyl-9,9'-spirobi[fluorene]

2-POPh ${ }_{2}$-SBF: diphenyl-9,9'-spirobi[9H-fluoren]-2-yl- Phosphine oxide

2-4Py-SBF: 4-(9,9'-spirobi[fluoren]-2-yl)pyridine

2-5Pm-SBF: 5-(9,9'-spirobi[fluoren]-2-yl)pyrimidine

2,2'-(SBF): 2,2"-Bi-9,9'-spirobi[9H-fluorene]

$\mathbf{2 , 2}$ '-(SBF) 3 : 2,2":2'", 2"''-Ter-9,9'-spirobi[9H-fluorene]

2,7,4'-(SBF) $)_{3}: 2,7-d i(9,9 '$ 'spirofluorenyl-4-yl)-9,9'-spirobifluorene

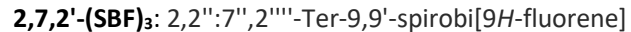

2,7,2'-(SBF) $4: 2,2^{\prime \prime}: 7^{\prime \prime}, 2^{\prime \prime \prime '}: 7^{\prime \prime \prime \prime, 2 " ' " '-Q u a t e r-9,9 '-s p i r o b i[9 H-f l u o r e n e] ~}$

4-AP-SBF:

4-Br-SBF: 4-bromo-9,9'-spirobifluorene

4,4'-Br 2 -SBF: 4,4'-dibromo-9,9'-Spirobi[9H-fluorene]

4-DBF-SBF: 4-(9,9'-spirobi[9H-fluoren]-4-yl)-dibenzofuran

4-DBT-SBF: 4-(9,9'-spirobi[9H-fluoren]-4-yl)-dibenzothiophene

4-EPHDB-SBF:

4-EPHDBA-SBF:

4,4'-FO-SBF: 4-(9,9'-spirobi[9H-fluoren]-4-yl)- 9H-Fluoren-9-one

4,4'-Pin 2 -SBF: 2,2'-(9,9'-spirobi[9H-fluorene]-4,4'-diyl)bis[4,4,5,5-tetramethyl-1,3,2-Dioxaborolane]

4-Pin-SBF: 2-(9,9'-spirobi[fluoren]-4-yl)-4,4,5,5-tetramethyl-1,3,2-dioxaborolane

4-Ph-SBF: 4-phenyl-9,9'-spirobi[fluorene]

4-PhCBz-SBF: 4-N-phenyl-carbazole-9,9'-spirobi[fluorene]

4-PhOMe 3 -SBF: 4-(3,4,5-trimethoxyphenyl)-9,9'-spirobi[fluorene]

4-POPh ${ }_{2}$-SBF: diphenyl-9,9'-spirobi[9H-fluoren]-4-yl-Phosphine oxide

4-4Py-SBF: 4-(9,9'-spirobi[fluoren]-4-yl)pyridine

4-3Py-SBF: 3-(9,9'-spirobi[fluoren]-4-yl)pyridine

4-2Py-SBF: 2-(9,9'-spirobi[fluoren]-4-yl)pyridine

4-5Pm-SBF: 5-(9,9'-spirobi[fluoren]-4-yl)pyrimidine

4-SFA-SFA: 10,10'-diphenyl-4',4"-bispiro[acridine-9(10H),9'-[9H]fluorene]

4-SFA-SBF: 10 -phenyl-4'-(9,9'-spirobi[9H-fluoren]-4-yl)-spiro[acridine-9(10H),9'-[9H]fluorene]

4,4'-(SBF) 2: 4-(9,9'-spirofluorenyl-4-yl)-9,9'-spirobifluorene

4,3'-(SBF) $2: 3,4^{\prime}-B i-9,9 '-$-spirobi[9H-fluorene]

4,4'-(SBF) $3: 4,4^{4}: 4^{\prime \prime \prime}, 4^{\prime \prime \prime '-T e r-9,9 '-s p i r o b i[9 H-f l u o r e n e] ~}$

4,4'-(SBF) $4: 4,4^{\prime \prime}: 4$ '", 4 "'"':4'"'", 4 '"'"'-Quater-9,9'-spirobi[9H-fluorene]

4,4'-(SBF) $5: 4,4 ": 4^{\prime \prime \prime, 4 " ' ": 4 ' " ' ", 4 ' " ' " ': 4 " ' " ' " ', 4 " ' " ' " '-Q u i n q u e-9,9 '-s p i r o b i[9 H-f l u o r e n e] ~}$

4-T-DKPP-SBF 2 :

4-VCbz-SBF:

4-VTPA-SBF:

4-VTPA-SBF(t-Bu)

AcOH: acetic acid

BD-1: diphenyl-[4-(2-[1,1';4',1"]terphenyl-4-yl-vinyl)-phenyl]-amine

BSFM : bis-9,9'-spirobi[fluoren-2-yl]-methanone

(Btp) $2 \mathrm{rr}(\mathrm{acac})$ : bis[2-(20-benzo[4,5-a]thienyl)pyridinato- $\mathrm{N}, \mathrm{C} 30]$ iridium(acetylacetonate)

CE: Current efficiency (in OLED or PhOLED)

CuPc: copper phthalocyanine

DCM: dichloromethane

$\Delta \mathrm{E}^{\mathrm{el}}$ : electrochemical band gap

$\Delta \mathrm{E}^{\mathrm{opt}}$ : optical band gap

DMF: $N, N$-Dimethylformamide

DNTPD: N,N'-diphenyl-N,N'-bis-[4-(phenyl-m-tolyl-amino)-phenyl]-biphenyl-4,4'-diamine

\section{DSBF-PA:}

DSC: differential scanning calorimetry

EML: emitting layer (in OLED or PhOLED)

EQE: external quantum efficiency (in OLED or PhOLED)

$\mathrm{E}_{\mathrm{T}}$ : triplet energy

FIrpic: Bis[2-(4,6-difluorophenyl)pyridinato- $\left.\mathrm{C}^{2}, N\right]$ (picolinato)iridium(III)

HAT-CN: Dipyrazino[2,3-f:2', 3'-h]quinoxaline 2,3,6,7,10,11-hexacarbonitrile

HOMO: Highest occupied molecular orbital

HTM: hole blocking material

$\operatorname{Ir}(\text { ppy })_{3}:$ Tris $\left[2\right.$-phenylpyridinato- $\left.\mathrm{C}^{2}, N\right]$ iridium(III)

ITO: Indium-Tin oxide

$\mathrm{K}_{2} \mathrm{CO}_{3}$ : potassium carbonate

$\mathrm{k}_{\mathrm{nr}}$ : non radiative rate constant

$\mathrm{k}_{\mathrm{r}}$ : radiative rate constant

Liq: 8-Hydroxyquinolinolato-lithium

LUMO: lowest unoccupied molecular orbital 
MADN: 2-methyl-9,10-di(2-naphthyl)anthracene

2-MeTHF: 2-Methyltetrahydrofuran

$\mathrm{MoO}_{3}$ : Molybdenum trioxide

NPB: N'-di(1-naphtyl)-N,N'-diphenylbenzidine

(ppq) 2 Ir(acac): bis(2,4-diphenylquinolyl- $N, C 2$ ) iridium (acetylacetonate)

$\mathrm{MsOH}$ : methanesulfonic acid

n-BuLi: n-Butyllithium

OLED: organic light-emitting diode

$\mathrm{P}(\mathrm{Cy})_{3}$ : Tricyclohexylphosphine

$\mathrm{Pd}_{2} \mathrm{dba}_{3}$ : Tris(dibenzylideneacetone)dipalladium(0)

$\mathrm{Pd}(\mathrm{dppf}) \mathrm{Cl}_{2}$ : [1,1'-Bis(diphenylphosphino)ferrocene]dichloropalladium(II)

$\mathrm{Pd}(\mathrm{OAc})_{2}$ : Palladium(II) acetate

$\mathrm{Pd}\left(\mathrm{PPh}_{3}\right)_{4}$ : Tetrakis(triphenylphosphine)palladium(0)

PE: power efficiency (in OLED or PhOLED)

PHC: pure hydrocarbon

Ph 2 Br: 2-bromo-biphenyl

PhOLED: phosphorescent organic ligth-emitting diode

$(\mathrm{ppq})_{2} \operatorname{Ir}(\mathrm{acac})$ : bis(2,4-diphenylquinolyl- $\mathrm{N}, \mathrm{C2}$ ') iridium(acetylacetonate)

$\mathrm{P}(\mathrm{t}-\mathrm{Bu})_{3}$ : Tri-tert-butylphosphine

$\Phi_{\text {sol: }}$ quantum yield

SBF: 9,9'-spirobifluorene

SBF: spirobifluorene

Spiro-2CBP: 2,7-Bis(carbazol-9-yl)-9,9-spirobifluorene

Spiro-OMeTAD : 2,2',7,7'-Tetrakis[N,N-di(4-methoxyphenyl)amino]-9,9'-spirobifluorene

TAPC: Di-[4-(N,N-di-p-tolyl-amino)-phenyl]cyclohexane

$T_{d}$ : decomposition temperature

$\mathrm{T}_{\mathrm{g}}$ : glass transition temperature

TGA: thermogravimetric analysis

TCTA: Tris(4-carbazoyl-9-ylphenyl)amine

THF: tetrahydrofuran

TMEDA: Tetramethylethylenediamine

TmPyPY: 1,3,5-Tri[(3-pyridyl)-phen-3-yl]benzene

TPBi: 2,2',2"-(1,3,5-Benzinetriyl)-tris(1-phenyl-1- $H$-benzimidazole)

TSPO1: diphenylphosphine oxide-4-(triphenylsilyl)phenyl

$\mathrm{V}_{\text {on }}$ : threshold voltage (in OLED or PhOLED) 


\section{REFERENCES}

[1] A. F. d. l'éclairage, 17, rue de l'Amiral Hamelin 75783 Paris Cedex 16.

[2] aG. ZISSIS, J.-J. DAMELINCOURT, Technique de l'Ingénieur 2004; bG. ZISSIS, X. DE LOGIVIERE, Technique de l'Ingénieur 2011.

[3] J. H. Seo, N. S. Han, H. S. Shim, J. H. Kwon, J. K. Song, Bull. Korean Chem. Soc. 2011, 32, 1415.

[4] E. Baranoff, B. F. E. Curchod, Dalton Trans. 2015, 44, 8318-8329.

[5] aH. Sasabe, J. Kido, Eur. J. Org. Chem. 2013, 2013, 7653-7663; bL. Xiao, Z. Chen, B. Qu, J. Luo, S. Kong, Q. Cong, J. Kido, Adv. Mater. 2011, 23, 926-952; cY. Tao, C. Yang, J. Qin, Chem. Soc. Rev. 2011, 40, 2943-2970.

[6] aC.-L. Ho, W.-Y. Wong, New J. Chem. 2013, 37, 1665-1683; bK. S. Yook, J. Y. Lee, Adv. Mater. 2012, 24, 3169-3190.

[7] aT. P. I. Saragi, T. Spehr, A. Siebert, T. Fuhrmann-Lieker, J. Salbeck, Chem. Rev. 2007, 107, 1011-1065; bR. Pudzich, T. Fuhrmann-Lieker, J. Salbeck, Adv. Polym. Sci. 2006, 199, 83-142.

[8] H. Schenk, Acta Crystallogr., Sect.B: Struct. Crystallogr. Cryst. Chem. 1972, $28,625$.

[9] J. Pei, J. Ni, X.-H. Zhou, X.-Y. Cao, Y.-H. Lai, J. Org. Chem. 2002, 67, 4924-4936.

[10] N. A. van Dantzig, D. H. Levy, C. Vigo, P. Piotrowiak, J. Chem. Phys. 1995, 103, 4894-4906.

[11] Z. Jiang, H. Yao, Z. Zhang, C. Yang, Z. Liu, Y. Tao, J. Qin, D. Ma, Org. Lett. 2009, 11, 2607-2610.

[12] S. Thiery, D. Tondelier, C. Declairieux, B. Geffroy, O. Jeannin, R. Métivier, J. RaultBerthelot, C. Poriel, J. Phys. Chem. C 2015, 119, 5790-5805.

[13] S. Thiery, C. Declairieux, D. Tondelier, G. Seo, B. Geffroy, O. Jeannin, R. Métivier, J. Rault-Berthelot, C. Poriel, Tetrahedron 2014, 70, 6337-6351.

[14] S. Thiery, D. Tondelier, C. Declairieux, G. Seo, B. Geffroy, O. Jeannin, J. RaultBerthelot, R. Metivier, C. Poriel, J. Mater. Chem. C 2014, 2, 4156-4166.

[15] L.-S. Cui, Y.-M. Xie, Y.-K. Wang, C. Zhong, Y.-L. Deng, X.-Y. Liu, Z.-Q. Jiang, L.S. Liao, Adv. Mater. 2015, 27, 4213-4217.

[16] C. Fan, Y. Chen, P. Gan, C. Yang, C. Zhong, J. Qin, D. Ma, Org. Lett. $\quad$ 2010, 12, 5648-5651.

[17] K.-T. Wong, Y.-L. Liao, Y.-T. Lin, H.-C. Su, C.-c. Wu, Org. Lett. 2005, 7, 51315134.

[18] X. Zhou, J. He, L. S. Liao, M. Lu, X. M. Ding, X. Y. Hou, X. M. Zhang, X. Q. He, S. T. Lee, Adv. Mater. 2000, 12, 265-269.

[19] C.-C. Wu, T.-L. Liu, Y.-T. Lin, W.-Y. Hung, T.-H. Ke, K.-T. Wong, T.-C. Chao, Applied Physic Letters 2004, 85, 1172-1174.

[20] S. E. Jang, C. W. Joo, J. S. Ok, Y. K. Soo, J. Y. Lee, Org. Electron. 2010, 11, 10591065.

[21] S. O. Jeon, K. S. Yook, C. W. Joo, H. S. Son, J. Y. Lee, Thin Solid Films 2010, 518, 3716-3720.

[22] S. Thiery, D. Tondelier, B. Geffroy, E. Jacques, M. Robin, R. Métivier, O. Jeannin, J. Rault-Berthelot, C. Poriel, Org. Lett. 2015, 17, 4682-4685.

[23] S.-C. Dong, C.-H. Gao, Z.-H. Zhang, Z.-Q. Jiang, S.-T. Lee, L.-S. Liao, Phys. Chem. Chem. Phys. 2012, 14, 14224-14228.

[24] S.-C. Dong, C.-H. Gao, X.-D. Yuan, L.-S. Cui, Z.-Q. Jiang, S.-T. Lee, L.-S. Liao, Org. Electron. 2013, 14, 902-908. 
[25] T. Liu, H. Sun, C. Fan, D. Ma, C. Zhong, C. Yang, Org. Electron. 2014, 15, 35683576.

[26] C. Quinton, S. Thiery, O. Jeannin, D. Tondelier, B. Geffroy, J. Rault-Berthelot, C. Poriel, ACS Appl. Mater. Interfaces 2017, submitted.

[27] I. Bulut, P. Chavez, S. Fall, S. Mery, B. Heinrich, J. Rault-Berthelot, C. Poriel, P. Leveque, N. Leclerc, RSC Adv. 2016, 6, 25952-25959.

[28] S. E. Jang, C. W. Joo, K. S. Yook, J.-W. Kim, C.-W. Lee, J. Y. Lee, Synth. Met. 2010, 160, 1184-1188.

[29] S. Lee, B. Kim, H. Jung, H. Shin, H. Lee, J. Lee, J. Park, Dyes Pigm. 2017, 136, 255261.

[30] S. Thiery, B. Heinrich, B. Donnio, C. Poriel, F. Camerel, J. Phys. Chem. C 2015, 119, 10564-10575.

[31] K. H. Lee, S. O. Kim, K. S. Yook, S. O. Jeon, J. Y. Lee, S. S. Yoon, Synth. Met. 2011, 161, 2024-2030.

[32] K. H. Lee, S. O. Kim, J. N. You, S. Kang, J. Y. Lee, K. S. Yook, S. O. Jeon, J. Y. Lee, S. S. Yoon, J. Mater. Chem. 2012, 22, 5145-5154.

[33] G. Valchanov, A. Ivanova, A. Tadjer, D. Chercka, M. Baumgarten, Org. Electron. 2013, 14, 2727-2736.

[34] aC. Adachi, R. C. Kwong, P. Djurovich, V. Adamovich, M. A. Baldo, M. E. Thompson, S. R. Forrest, Appl. Phys. Lett. 2001, 79, 2082-2084; bN. S. Han, S. H. Sohn, S. M. Park, J. K. Song, Bull. Korean Chem. Soc. $\quad$ 2013, 34, 1547-1550.

[35] C. W. Lee, J. Y. Lee, Chem. Mater. 2014, 26, 1616-1621. 